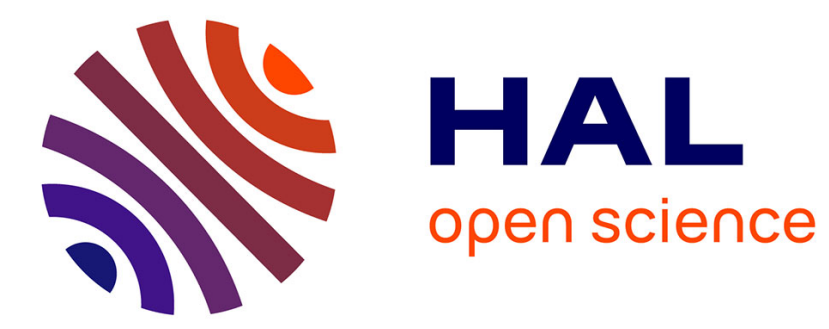

\title{
All-experimental analysis of doubly resonant Sum-Frequency Generation spectra: application to aggregated rhodamine films
}

Bertrand Busson, Maissa Farhat, Paul-Jérémy Nini Teunda, Sandra Roy, Tasha Jarisz, Dennis K Hore

\section{To cite this version:}

Bertrand Busson, Maissa Farhat, Paul-Jérémy Nini Teunda, Sandra Roy, Tasha Jarisz, et al.. Allexperimental analysis of doubly resonant Sum-Frequency Generation spectra: application to aggregated rhodamine films. Journal of Chemical Physics, 2021, 154, pp.224704. 10.1063/5.0048787 . hal-03254451

\section{HAL Id: hal-03254451 \\ https://hal.science/hal-03254451}

Submitted on 8 Jun 2021

HAL is a multi-disciplinary open access archive for the deposit and dissemination of scientific research documents, whether they are published or not. The documents may come from teaching and research institutions in France or abroad, or from public or private research centers.
L'archive ouverte pluridisciplinaire HAL, est destinée au dépôt et à la diffusion de documents scientifiques de niveau recherche, publiés ou non, émanant des établissements d'enseignement et de recherche français ou étrangers, des laboratoires publics ou privés. 


\title{
All-experimental analysis of doubly resonant Sum-Frequency Generation spectra: application to aggregated rhodamine films
}

\author{
Bertrand Busson, ${ }^{1}$ Maissa Farhat,,${ }^{1}$ Paul-Jérémy Nini Teunda, ${ }^{1}$ Sandra Roy, ${ }^{2}$ Tasha \\ Jarisz, ${ }^{2}$ and Dennis K. Hore ${ }^{2,3}$ \\ 1) Université Paris-Saclay, CNRS, Institut de Chimie Physique, UMR 8000, \\ 91405 ORSAY, France ${ }^{\mathrm{a})}$ \\ ${ }^{2)}$ Department of Chemistry, University of Victoria, Victoria, British Columbia, \\ V8W 3V6, Canada \\ 3) Department of Computer Science, University of Victoria, Victoria, \\ British Columbia, V8W 3P6, Canada ${ }^{\mathrm{b}}$
}

(Dated: 27 April 2021)

A new method is proposed to analyze doubly-resonant infrared-visible sum-frequency (DR-SFG) spectra. Based on the transform technique, this approach is free from assumptions about vibronic modes, energies, or line widths, and accurately captures through the overlap spectral function all required aspects of the vibronic structure from simple experimental linear absorption spectra. Details and implementation of the method are provided, along with three examples treating rhodamine thin films about one monolayer thick. The technique leads to a perfect agreement between experiment and simulations of the visible DR-SFG lineshapes, even in the case of complex intermolecular interactions resulting from J-aggregated chromophores in heterogeneous films. For films with mixed $\mathrm{H}$ - and J-aggregates, separation of their responses shows that the J-aggregate DR-SFG response is dominant. Our analysis also accounts for the unexplained results published in the early times of DR-SFG experiments.

\footnotetext{
${ }^{a)}$ Electronic mail: bertrand.busson@universite-paris-saclay.fr

b) Electronic mail: dkhore@uvic.ca
} 


\section{INTRODUCTION}

The potential for solar cells as renewable energy sources is increasingly recognized as better devices come to the market. The use of organic dyes as sensitizers is one of the promising routes to enhance their performance. As a result, there has been an increased effort to develop new dye-sensitized materials for more efficient light harvesting, ${ }^{1}$ aiming at becoming metal-free, ${ }^{2}$ and resting on the peculiar properties of big families of molecules like porphyrins, ${ }^{3-6}$ phtalocyanines,${ }^{7}$ triarylamines (D5 family), ${ }^{8-10}$ squaraines, ${ }^{11,12}$ ruthenium complexes (N3 family) ${ }^{13,14}$ or rhodamines, ${ }^{15}$ as sensitizers or co-sensitizers. ${ }^{16}$ The rhodamine family ${ }^{17}$ encompasses many chromophores with intense absorption and fluorescence properties spanning the visible range. In addition to their interest in device development, rhodamines have often been used as probes or benchmark molecules for new resonant optical spectroscopies in the visible, or to compare theoretical predictions to experiments in surface-enhanced ${ }^{18}$ (SERS) and tip-enhanced ${ }^{19}$ (TERS) Raman scattering, hyper-Raman scattering, ${ }^{20}$ surface enhanced hyper-Raman scattering, ${ }^{21,22}$ twophoton fluorescence, ${ }^{23}$ hyper-Rayleigh scattering, ${ }^{24}$ stimulated Raman spectroscopy, ${ }^{25}$ single molecule SERS ${ }^{26}$ and TERS, ${ }^{27}$ and plasmonic coupling. ${ }^{28}$ As for second order nonlinear optics, rhodamines have been choice molecules for studying the structure, aggregation properties and optical response of adsorbed thin films using second harmonic generation ${ }^{29}$ (SHG) and sum-frequency generation $(\mathrm{SFG}),{ }^{30-32}$ even before the measurement of the first SFG photon. ${ }^{33}$

In order to optimize the conversion of solar energy into electricity, the interaction between the dyes and the surface of the photo-electrode must be optimized. From a structural point of view, the surface orientation, ${ }^{4}$ electronic structure ${ }^{34}$ and aggregation state ${ }^{15}$ of the dye molecules are critical aspects of their performance, and must be precisely known and controlled in situ. ${ }^{12,35}$ Furthermore, these effects are coupled, as aggregation often leads to a change in the molecular tilt angle and modifies the electronic states. Surface orientation controls the efficiency of light harvesting and optimized electron injection into the photoelectrode through a donor group. ${ }^{36}$ The optical absorption range and efficiency critically depends on the local electronic structure of the dyes after immobilization, which may be modified by the presence of the substrate and by molecular aggregation. For example, the formation of $\mathrm{H}-$ and $\mathrm{J}$-aggregates ${ }^{37}$ (wherein transition dipoles couple in a parallel and 
head-to-tail geometry, respectively ${ }^{29}$ ) induces a blueshift and a redshift, respectively, of the absorption maximum, and may lead to either enhanced ${ }^{11,15,38,39}$ or decreased $^{35}$ photovoltaic $^{2}$ performance (see Ref. 32 for a review). Concerning the electron transfer dynamics, it has been shown that vibrations play a key role in this injection process, either in the ground state $^{40}$ or the excited state. For the latter, after optical excitation of the system and creation of the electron-hole pair in the donor area, efficient electron transfer from the donor state to the acceptor state (or to the substrate) is achieved by an overlap of the orbitals of the two states involved and a delocalization of the electron wavefunction across the bridge between the two entities. ${ }^{41}$ Experimental evidence show that there is a vibrational component to the charge transfer mechanism. ${ }^{40}$ Theoretical calculations support the idea that the ultrafast dynamics of the vibrational wavepacket in the molecular excited state (i.e. the vibronic wavepacket) is vital to promote or quench the transfer. ${ }^{42}$ More precisely, the motions induced by the excitations of one or two vibrational modes promote the orbital overlap and help bridge the small energy gap between donor and acceptor states. ${ }^{43}$

Experimental characterization of the static and dynamic processes in situ requires specific adapted investigation techniques. In addition to the standard electrical, photochemical, photoelectrochemical and linear optical tools used to determine and control the efficiency of the photovoltaic process,${ }^{1}$ Doubly Resonant Sum-Frequency Generation spectroscopy (DRSFG) may enable understanding at the molecular scale the phenomena taking place at the molecule-substrate junction. It is well-known that vibrational (i.e. singly-resonant, SR) infrared-visible SFG is intrinsically sensitive to molecules at an interface, and allows information about their orientation to be extracted from their vibrational modes, through polarization combinations $s^{4,44}$ and/or peak intensity ratios $^{45}$ for example. In addition to vibrations, DR-SFG resonantly couples light to electronic and vibronic molecular transitions, spectroscopically resolving the optical excitations in the visible range in addition to the infrared (IR), ${ }^{30}$ making it sensitive to any evolution of the electronic structure upon adsorption, through aggregation in particular. ${ }^{32}$ Finally, SFG gives access to ultrafast vibrational dynamics in molecules ${ }^{46}$ and, as we will explain below, the DR-SFG spectroscopic response in the visible is strongly related to the excited state vibrational wavepacket dynamics through the overlap spectral function. ${ }^{47,48}$ For all these reasons, DR-SFG appears suitable to complement the panel of optical spectroscopic techniques applied to study charge transfer processes in situ, from static absorption and resonance Raman to ultrafast pump-probe and 
two-dimensional electronic ${ }^{40}$ and infrared spectroscopies.

Historically, DR-SFG with tunable visible lasers has been applied to investigate the model chiral response of coupled oscillators ${ }^{49}$ like binapthol in the liquid phase $\mathrm{e}^{50-52}$ or in monolayers ${ }^{53-55}$ both theroretically and experimentally; the vibrational and electronic structures of chromophores ${ }^{56-60}$ and conjugated polymers; ${ }^{61,62}$ and the charge transfer states induced by molecular adsorption on metals. ${ }^{63-69}$ When the visible wavelength is tuned, most of these works also confront the delicate problem of data analysis, and coupling between electronic and vibrational resonances. The latter makes the former much more complicated than in the singly IR-resonant case because the whole molecular vibronic structure is now involved, as is explained below. In the first publication showing experimental DR-SFG spectra of rhodamine $6 \mathrm{G}$, Raschke et al. noted that the excitation spectra in the visible (i.e. the evolution of the amplitudes of the vibration modes as a function of the visible wavelength) comprised only one peak instead of the two expected peaks, leading them to conclude that there was no contribution from higher order vibronic transitions to their DRSFG spectra because their frequency widths were much broader than for the $0-0$ line. ${ }^{30}$ This conclusion is also used in subsequent publications and leads several authors to simplify the DR-SFG formulas by truncating the vibronic summations to the lowest order and/or to the IR-excited mode only, ${ }^{61}$ which is one of the strategies encountered in the literature for an easy interpretation of DR-SFG spectra. The second major strategy consists of calculating the electronic response and vibration mode activities through first principle methods (e.g. DFT/TD-DFT, CCSD) to predict the DR-SFG spectra. ${ }^{60}$ Of course, this method supposes that the molecules keep their lower energy conformation, usually calculated in the gas phase, and do not aggregate when adsorbed on the surface. ${ }^{31}$

In order to avoid these approximations and restrictions when modeling the electronic part of the DR-SFG response, we propose an alternate strategy which naturally takes the full vibronic structure into account. The ultimate goal is to determine all unknown relevant parameters through experiments with minimal hypotheses and approximations. The first step, explored here using the example of rhodamine 123 and rhodamine $6 \mathrm{G}$ films on glass, consists of simulating the excitation profiles of the vibration modes using only experimental data. Following the original idea of the transform technique, we embed the vibronic sum-overstates summations into a quantity (the overlap spectral function $\Phi$ ) directly deduced from the experimental absorption spectrum. We may avoid the approximations usually employed 
for the direct sum-over-states procedure, as all vibronic terms are readily included in $\Phi$. This method also does not require any first principle calculation or hypothesis on the molecular structure, and allows straightforward embedding of aggregation and inhomogeneous broadening effects.

From a more general perspective, the idea of the transform technique has never been used for the analysis of DR-SFG data. We note that a parallel approach is developed in Ref. 60, except that the energy lineshapes are computed, as the vibrational properties, from first principle methods. ${ }^{70,71}$

\section{THEORETICAL BACKGROUND}

\section{A. Double resonance SFG spectroscopy}

Sum-Frequency Generation is a second-order nonlinear optical process. Two laser beams (frequencies $\omega_{1}$ and $\left.\omega_{2}\right)$ interact with matter to create a nonlinear polarization $P^{(2)}\left(\omega_{\mathrm{SFG}}\right)$ oscillating and emitting radiation at frequency $\omega_{\mathrm{SFG}}=\omega_{1}+\omega_{2}$. The nonlinear polarization is proportional to the local electric field amplitudes $E^{1}$ and $E^{2}$ through their $(i, j, k)$ Cartesian components in the laboratory frame $P_{i}^{(2)}\left(\omega_{\mathrm{SFG}}\right)=\chi_{i j k}^{(2)} E_{j}^{1}\left(\omega_{1}\right) E_{k}^{2}\left(\omega_{2}\right)$, and to the nonlinear susceptibility tensor $\chi^{(2)}$, characteristic of matter where the interaction takes place. This macroscopic quantity has the following properties: it vanishes upon centrosymmetry and consequently restricts the SFG process mostly to interfaces between centrosymmetric media; it becomes resonantly enhanced when one of the beams excites an allowed optical transition. As a consequence, SFG is often used as a spectroscopy in the infrared range, with $\omega_{2}=\omega_{\mathrm{IR}}$, to provide a vibrational fingerprint of interfaces. In this case, the surface nonlinear polarization $P^{(2)}$ radiates light to the far field, with an intensity proportional to the incoming visible and IR intensities, to the squared SFG frequency $\left(\omega_{\mathrm{SFG}}\right)^{2}$ and to the square modulus of $\chi^{(2)}$ corrected from local field effects. ${ }^{45}$ For molecules at an interface,

we have $\chi_{i j k}^{(2)}=N_{s} \beta_{i j k} / \epsilon_{0}$ where $N_{s}$ is the surface density of molecules and $\beta_{i j k}=\left\langle\beta_{\alpha \beta \gamma}\right\rangle_{i j k}$ is the molecular hyperpolarizability in the laboratory frame, deduced from the molecular frame (Cartesian coordinates: $\alpha, \beta, \gamma$ ) after averaging over molecular orientations. When applied to chromophores or optically active materials in the visible range, the process with $\omega_{1}=\omega_{\text {vis }}$ also becomes resonant with an electronic transition in the visible, and SFG is said 
doubly resonant. Considering the lifetimes of the vibrational and electronic excited states, IR-vis DR-SFG dominates over the vis-IR process. ${ }^{72}$ Following resonant annihilation of the IR photon, the visible annihilation and SFG creation processes may include all the allowed transitions involving any vibrational level of the excited state, i.e. the whole molecular vibronic structure (see Ref. 47 for a review). The vibronic structure is considered as a first approximation identical to the vibrational one, which is equivalent to considering only linear electron-vibration couplings; extension to quadratic coupling leading to mode distortion and Duschinsky rotation may be found in the literature ${ }^{47,73,74}$ but will not be considered here.

\section{B. Linking absorption and DR-SFG spectroscopy: the $\Phi(\omega)$ function}

Under the Born-Oppenheimer and harmonic oscillator approximations, for a molecule with a single active electronic transition, the molecular doubly resonant hyperpolarisability may be written as: ${ }^{47}$

$$
\beta_{\alpha \beta \gamma}=1 / \hbar^{2} \sum_{l}^{\mathrm{IR}-\text { active }} \frac{A_{l}^{\alpha \beta \gamma} D_{l}\left(\omega_{\mathrm{SFG}}\right)}{\omega_{\mathrm{IR}}-\omega_{l}+i \Gamma_{l}}
$$

where $A_{l}^{\alpha \beta \gamma}=\mu_{g \leftarrow e}^{\alpha} \mu_{e \leftarrow g}^{\beta}\left(\frac{\partial \mu^{\gamma}}{\partial Q_{l}}\right)_{0}$ is the static SFG amplitude and involves the dipole moment $\mu$ of the electronic transition between ground $(g)$ and excited $(e)$ states, and the vibrational transition moment $\left(\frac{\partial \mu^{\gamma}}{\partial Q_{l}}\right)_{0}$ of vibration mode $l$, where $Q_{l}$ is the normal mode coordinate of the vibration, projected onto a molecular frame. $D_{l}(\omega)$ is the excitation spectrum of mode $l$ and contains the processes resonant with the vibronic structure. For a Franck-Condon active mode, we have

$$
D_{l}\left(\omega_{\mathrm{SFG}}\right)=\frac{\Delta_{l}}{2}\left[\Phi\left(\omega_{\mathrm{SFG}}\right)-\Phi\left(\omega_{\mathrm{SFG}}-\omega_{l}\right)\right]
$$

and, in particular, at resonance with vibration mode $l\left(\omega_{\mathrm{IR}}=\omega_{l}\right)$ :

$$
D_{l}\left(\omega_{\mathrm{SFG}}\right)=\frac{\Delta_{l}}{2}\left[\Phi\left(\omega_{\mathrm{SFG}}\right)-\Phi\left(\omega_{\mathrm{vis}}\right)\right]
$$

where $\Delta_{l}$ is the shift in normal mode coordinates between the vibration potential energy curves of mode $l$ in the ground and excited states. For a Franck-Condon inactive mode, the DR-SFG process may exist through the Herzberg-Teller mechanism. ${ }^{31}$ We introduce the overlap spectral function ${ }^{48,74} \Phi(\omega)$, defined as the Fourier transform of the overlap between ground and excited states vibrational wavepackets, which accounts for the full sum-overstates of the vibronic structure and is common to all vibration modes $l$. Function $\Phi$ is the core 
of the transform technique, an alternative to sum-over-states methodology for data analysis in resonant Raman scattering. ${ }^{75,76}$ Once $\Phi$ is known, two parameters per vibration mode are enough to simulate the DR-SFG spectra as a function of the IR and visible wavenumbers, namely $A_{l}$ and $\Delta_{l}$. In particular, Eqs. 1 and 2 show that we may separate the amplitudes related to the relative orientations of light beams and molecules (through $\alpha, \beta$ and $\gamma$ ) from the resonant lineshapes depending on energies $\omega_{\mathrm{IR}}, \omega_{\mathrm{vis}}$ and $\omega_{\mathrm{SFG}}$. On vibrational resonance, the dispersion of the excitation profile as a function of the visible wavenumber solely depends on the $\Phi$ terms in Eq. 3. $\Phi(\omega)$ may be evaluated by expanding it into a sum-over-states formulation ${ }^{72,74,77}$ as sums of resonant Lorentzian functions, taking care of including all modes of the vibronic structure and avoiding truncation at low order, as is often done. ${ }^{61,62,64}$ The great advantage of this formulation is that function $\Phi$ may also be deduced from experiments. The simplest way is to consider the visible absorption spectrum, for which the absorbance $\mathrm{A}(\omega)$ may be written as:

$$
A(\omega) \propto \omega\left|\mu_{e \leftarrow g}\right|^{2} \operatorname{Im}[\Phi(\omega)]
$$

As $\Phi(\omega)$ is only an energy lineshape, the choice of polarization and angle of incidence for the absorbed light will only affect the transition amplitude $\mu_{e \leftarrow g}$ and leave $\Phi$ unchanged. It is essential to measure the absorbance on the same interface as the DR-SFG spectrum itself, i.e. at the monolayer level. As a matter of fact, when considering molecular layers adsorbed on a substrate, adsorption may modify the molecular electronic properties through dimerization, aggregation, charge transfer from the substrate or chemical reaction.

\section{Reconstructing $\Phi(\omega)$ from experimental data}

As $\Phi(\omega)$ essentially consists of sums of complex Lorentzian functions, each one accounting for an individual jump from the ground state to one of the states composing the vibronic structure, it must fulfill the Kramers-Kronig (KK) relationships. In the classical transform technique for resonant Raman scattering, the real part of the complex function $\Phi$ is therefore recovered by a KK transformation of its imaginary part: ${ }^{7-85}$

$$
\operatorname{Re}[\Phi(\omega)]=\frac{1}{\pi} \int_{-\infty}^{+\infty} \frac{\operatorname{Im}[\Phi(\Omega)]}{\Omega-\omega} d \Omega
$$

where the principal value for the integral is implied to avoid convergence problems. As pointed out in Ref. 85, the KK method suffers from shortcomings (the imaginary part must 
be set to zero outside the peak region, numerical noise appears from integration, convergence problems must be taken care of). As we will see below, it is also not flexible enough to handle more complex situations, for example when two kinds of aggregates coexist. Following Ref. 85, we therefore propose to determine the real part by curve fitting of the imaginary part. The absorbance features of a chromophore in solution or as a monolayer (see for example Fig. 2 (A)) have a reproducible structure: they consist of a main peak, related to the $0-0$ electronic transition (vibrationless frequency $\omega_{0}$, width $\Gamma_{0}$ ), completed on the high energy side by a vibronic tail summing up all the allowed vibronic transitions. ${ }^{74}$ In condensed matter, the widths of all the resonant features are the result of a convolution between the natural width $\Gamma$, related to the Lorentzian nature of the resonance and to the lifetimes of the transitions, and an inhomogeneous broadening, related to the coexistence of various local environments and interactions for each molecule, slightly modifying individual electronic states and efficiently modeled by a Gaussian distribution. In other words, the imaginary part of $\Phi$ is a sum of Voigt profiles. ${ }^{86}$ Turning back to the full complex $\Phi(\omega)$ function, it is therefore also possible to model it as the sum of two complex contributions. ${ }^{85}$ On one side, a Faddeeva function ${ }^{87}$ represents the inhomogeneously broadened 0-0 line, centered on $\omega_{0}$, with Lorentzian width $\Gamma_{0}$ and Gaussian width $\sigma_{0}$. On the other side, we consider the vibronic tail as a sum of individual Lorentzian functions resonant with a vibronic energy and weighted by a transition probability (related to the Franck-Condon or Herzberg-Teller nature of the vibration mode and its vibrational overlap). We know that these probabilities decrease towards high energies as they involve overtones and combination bands; at low energies, the start of the vibronic tail overlaps with the central peak. As a consequence, in condensed matter, the vibronic tail displays a maximum in the fingerprint region (at energy $\left.\omega_{0}+\omega_{c}\right)$ and appears as a continuum of Lorentzian contributions distributed around it. For organic chromophores, considering the high density of vibration modes involved in the vibronic structure, it is reasonable to model it by a continuous Gaussian distribution of vibronic modes, and consequently the full vibronic tail by a second Faddeeva function, centered on $\omega_{0}+\omega_{c}$, with Lorentzian width $\Gamma_{\text {vib }}$ and Gaussian width $\sigma_{\text {vib }}$. The total fit function is therefore:

$$
\operatorname{Im}[\Phi(\omega)]=\operatorname{Im}\left[\int_{-\infty}^{+\infty} \frac{A_{0-0} G_{0-0}\left(\Omega_{0}, \sigma_{0}\right)}{\omega-\Omega_{0}+i \Gamma_{0}} d \Omega_{0}+\int_{-\infty}^{+\infty} \frac{A_{\mathrm{vib}} G_{\mathrm{vib}}\left(\Omega_{\mathrm{vib}}, \sigma_{\mathrm{vib}}\right)}{\omega-\Omega_{\mathrm{vib}}+i \Gamma_{\mathrm{vib}}} d \Omega_{\mathrm{vib}}\right]
$$


where

$$
G_{0-0}\left(\Omega_{0}, \sigma_{0}\right)=\frac{1}{\sigma_{0} \sqrt{\pi}} \exp \left[-\left(\frac{\omega_{0}-\Omega_{0}}{\sigma_{0}}\right)^{2}\right]
$$

and

$$
G_{\mathrm{vib}}\left(\Omega_{\mathrm{vib}}, \sigma_{\mathrm{vib}}\right)=\frac{1}{\sigma_{\mathrm{vib}} \sqrt{\pi}} \exp \left[-\left(\frac{\omega_{0}+\omega_{c}-\Omega_{\mathrm{vib}}}{\sigma_{\mathrm{vib}}}\right)^{2}\right]
$$

from which the real part is easily deduced.

\section{Multiple excited states}

When several electronic resonances compete for the DR-SFG enhancement, giving rise to two or more resonances in the absorption spectrum, the analysis complexifies. We suppose that two distinct excited states (labelled $\mathrm{H}$ and $\mathrm{J}$ in reference to the two aggregation states of rhodamines $^{37}$ ), coexisting at the molecular level, contribute to the optical processes involved in the resonant absorption and DR-SFG processes. Each state has its own vibronic structure, represented by a distinct $\Phi_{H / J}$ function. In the absorption spectrum, their contributions simply sum up, weighted by population factors $P_{H}$ and $\left(1-P_{H}\right)$ :

$$
A(\omega) / \omega \propto P_{H}\left|\mu_{e \leftarrow g, H}\right|^{2} \operatorname{Im}\left[\Phi_{H}(\omega)\right]+\left(1-P_{H}\right)\left|\mu_{e \leftarrow g, J}\right|^{2} \operatorname{Im}\left[\Phi_{J}(\omega)\right]
$$

The situation becomes more complex for the DR-SFG response. As H and J states proceed from the same molecular monomers, they share the same vibrational levels in the ground state. However, from Eq. 1, the excitation profile of mode $l$ becomes:

$$
P_{H} A_{l, H}^{\alpha \beta \gamma} D_{l, H}+\left(1-P_{H}\right) A_{l, J}^{\alpha \beta \gamma} D_{l, J}
$$

Functions $\Phi_{H}$ and $\Phi_{J}$ are therefore weighted by a product of factors $\mu_{H}^{\alpha} \mu_{H}^{\beta}\left(\frac{\partial \mu^{\gamma}}{\partial Q_{l}}\right)_{0, H} \Delta_{l, H}$ and $\mu_{J}^{\alpha} \mu_{J}^{\beta}\left(\frac{\partial \mu^{\gamma}}{\partial Q_{l}}\right)_{0, J} \Delta_{l, J}$ to generate the full excitation profile. As $\mathrm{H}$ - and J-aggregate geometries significantly differ, ${ }^{29}$ there is no direct relationship between these four quantities for one type of aggregate or the other: electronic transition dipole moments $\mu_{g \leftarrow e}$ depend on the interaction geometry between the molecules, and their projections vary accordingly; IR transition dipole moments $\left(\frac{\partial \mu^{\gamma}}{\partial Q_{l}}\right)_{0}$ depend on the orientation of the molecules in the aggregate relative to the surface; normal mode shifts $\Delta_{l}$ relate to the electronic structures of the excited states and therefore also depend on the nature of the aggregate. The last two in particular do not contribute to the absorption spectrum. Contrary to the single electronic 
excited states situation, there is now no direct proportionality between the complex lineshape functions of the absorption and DR-SFG excitation spectra. The effective $\Phi$ function that may be reconstructed from a KK-transformation of the normalized absorption spectrum (Eq. 9) does not match that giving rise to the DR-SFG excitation profiles in Eq. 10. The fitting procedure provides a route for overcoming this problem: after extracting the global $\operatorname{Im}[\Phi]$ function from the absorption spectrum, we may separate its $\mathrm{H}$ and $\mathrm{J}$ components by curve fitting, which also provides at the same time their real parts. From the simulated $\Phi_{H}(\omega)$ and $\Phi_{J}(\omega)$ functions, we may reconstruct the total lineshape of the DR-SFG excitation spectrum, determining in this way the balance between $\mathrm{H}$ and $\mathrm{J}$ contributions.

\section{EXPERIMENTAL METHODS}

Solutions of rhodamine 6G (Sigma-Aldrich) were prepared in absolute ethanol with various concentrations $\left(10^{-6}, 10^{-5}, 10^{-4}, 2 \times 10^{-4}, 5 \times 10^{-4}\right.$ and $\left.10^{-3} \mathrm{M}\right)$. For rhodamine 123 (Sigma-Aldrich), concentrations in ethanol were chosen as $10^{-4}$ and $10^{-3} \mathrm{M}$. Rhodamine films were prepared by spin coating on ultrasonically cleaned microscope glass substrates, then dried in air. For SFG experiments, the rotation speed was set to 2000 rotations per minute. The influence of the rotation speed on the film structure was studied by UV-visible absorption and vibrational SFG by comparing 2000 and $9000 \mathrm{rpm}$ R6G films.

UV-visible absorbance spectra were recorded at normal incidence on a Cary 5000 spectrometer (Agilent) with $1 \mathrm{~nm}$ resolution.

The doubly resonant SFG spectra were recorded on the previously described set-up at Institut de Chimie Physique in Orsay. ${ }^{88}$ Briefly, a pulsed $1064 \mathrm{~nm} \mathrm{Nd:YVO4} \mathrm{laser} \mathrm{(7.5} \mathrm{ps,}$ $62.5 \mathrm{MHz}$ ) is used, after temporal shaping in $2 \mu$ s long trains at $25 \mathrm{~Hz}$ and amplification, to synchronously pump two optical parametric oscillators (OPO) for variable frequency output. The IR-OPO is based on a $\mathrm{AgGaS}_{2}$ crystal and generates IR radiation in the $1200-2800 \mathrm{~cm}^{-1}$ range. The visible OPO is pumped by the third harmonic and generates visible colors tunable in the $420-710 \mathrm{~nm}$ range. The two beams are slightly focused on the sample surface with angles of incidence $55^{\circ}$ and $65^{\circ}$ for the visible and IR, respectively. SFG is measured in reflection after spatial and frequency filtering through a double grating monochromator (Acton Trivista). In order to work in the $1600 \mathrm{~cm}^{-1}$ region, the IR path is purged with dry air but still experiences water absorption on its way, leading to dips in the 

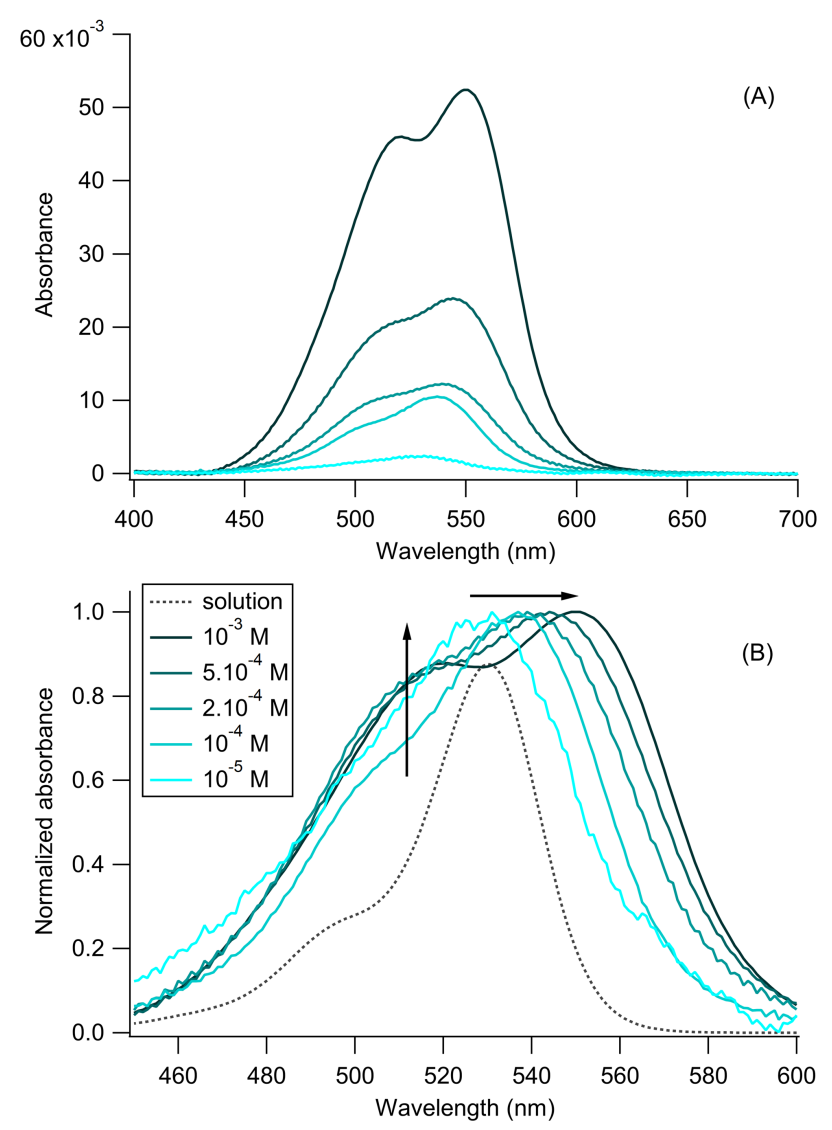

FIG. 1. Absorbance spectra (normal incidence) of rhodamine 6G films spin-coated on glass as a function of the solution concentration in ethanol. (A) Baseline-corrected spectra. (B) All spectra normalized to 1 . The arrows show the redshift of the J-aggregate and the increase of the $\mathrm{H}-$ aggregate peaks.

SFG spectra, corrected by post-processing and smoothing. The water absorption bands are used for an accurate IR frequency calibration by comparison to literature data. ${ }^{89}$

\section{STRUCTURE AND OPTICAL PROPERTIES OF THE RHODAMINE FILMS}

\section{A. Visible absorption spectra}

In order to have a precise knowledge of the structures of the rhodamine film on glass, we have studied the visible absorption spectra of R6G films with concentrations ranging from $10^{-6}$ to $10^{-3} \mathrm{M}$ (Fig. 1) at $9000 \mathrm{rpm}$. As studied by Chapman et al. ${ }^{37}$, the high and 
low rotation speeds do not induce significant changes in the visible absorbance lineshapes. Here we have focussed on the high speed data as they show clear aggregation trends as a function of rhodamine concentration. No signal could be measured from the $10^{-6} \mathrm{M}$ film. The absorption maxima increase with the concentration, which proves that deposition is a continuous process going beyond a monolayer. ${ }^{37}$ From Ref. 29, a monolayer is reached for peak absorbance around 0.02 , in our case around $5 \times 10^{-4} \mathrm{M}$, close to their value. However, this supposes that molecules do not aggregate on the films. As illustrated in Fig. 1, experience shows indeed a high degree of molecular aggregation, evidenced by the split of the monomer peak (represented by the solution curve, absorption maximum $530 \mathrm{~nm}$ ) into a blueshifted peak and a redshifted one, attributed to $\mathrm{H}$ - and J-aggregates, ${ }^{37,90}$ respectively. The magnitude of the redshift increases with concentration to reach $21 \mathrm{~nm}$ for $10^{-3} \mathrm{M}$. On the other hand, the amount of $\mathrm{H}$-aggregates increases faster with concentration than the J-aggregates, and their ratios stabilizes roughly after the monolayer level is reached. Using different substrates, some authors do not detect the J-aggregated peak. ${ }^{91}$ In Fig. 1B, the absorbance curve of the solution shows the vibronic tail as a high energy shoulder to the central peak, as no aggregation is expected in solution below $5 \times 10^{-3}$ M. ${ }^{90,92}$ Interpretation of the DR-SFG results relies, as explained above, on the in situ absorption spectra for the determination of the overlap spectral function.

In Fig. 2, we present the absorption spectra of the three films analyzed in the DR-SFG experiments below. The R6G film shows a clear presence of two maxima corresponding to the two aggregation geometries, as is directly deduced from the comparison to the above analysis. For the $10^{-3}$ M R123 film, the presence of two peaks is less obvious but the shape and width of the maximum, together with the comparison to the solution spectrum, show that there are indeed two electronic states involved, resonant with $\mathrm{H}$ - and J-aggregates. The situation is different for the $10^{-4} \mathrm{M}$ R123 film because the absorption spectrum shows only one resonance maximum, redshifted with respect to the solution peak, therefore indicating J-aggregation in the film. On the other hand, the peak is much broader than in solution, which could be attributed to either inhomogeneous broadening or the presence of a small contribution from $\mathrm{H}$-aggregates on the high energy side. Still the total width remains clearly smaller than for the $10^{-3} \mathrm{M}$ film, leading to favor the first hypothesis at this stage. 

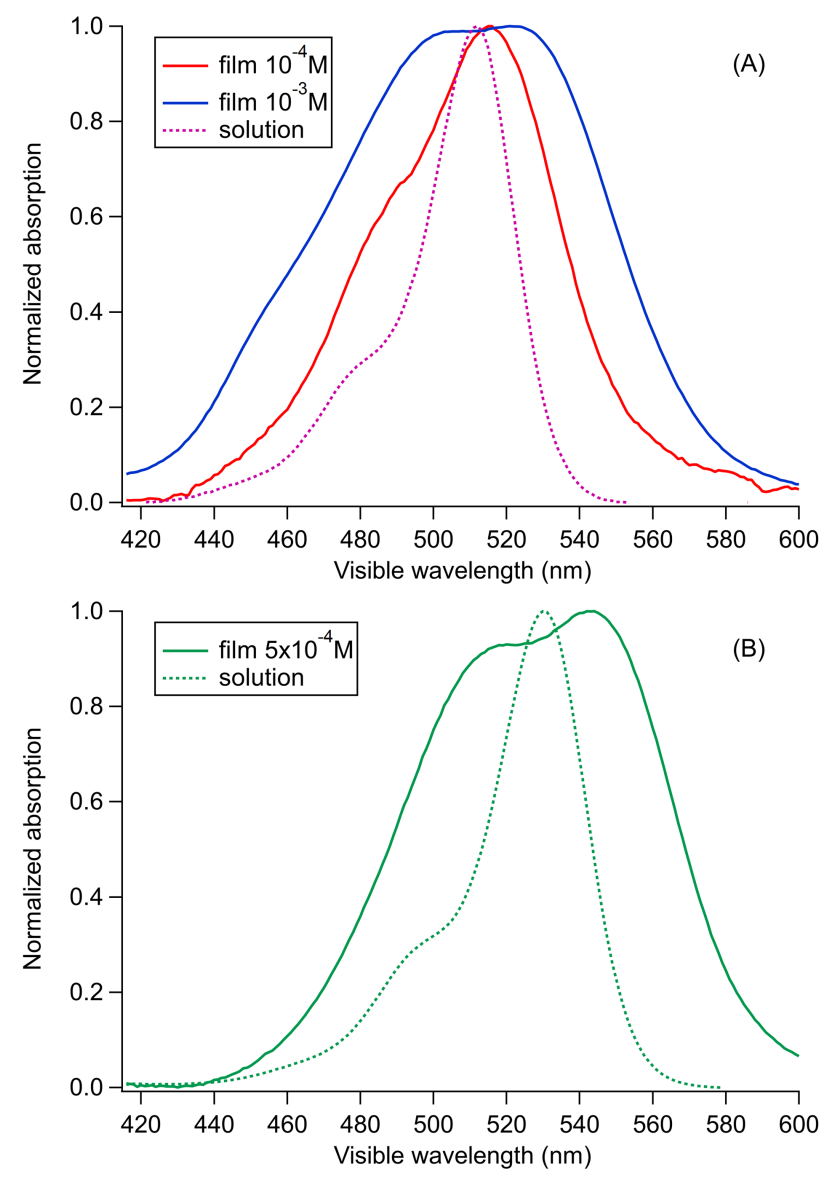

FIG. 2. Absorbance spectra (normal incidence, normalized to unity) of (A) R123 and (B) R6G films spin-coated on glass. Solution absorption spectra are superimposed (dotted lines) in order to evidence the redshift of the $\mathrm{J}$-aggregate peak and the presence of the $\mathrm{H}$-aggregate peak.

\section{B. Vibrational SFG spectra}

A full analysis of vibrational normal modes was performed in Ref. 93 and more thorough studies by DFT calculations, especially in the rich $1500-1700 \mathrm{~cm}^{-1}$ region. ${ }^{31,94}$ The SFG vibrational spectra, recorded in double resonance conditions for the SFG energy, show that the films are rather heterogeneous, with the SFG intensities depending on the point probed on the surface. It is therefore not possible to compare absolute SFG intensities for different films. In Fig. 3 we show the vibrational signatures of R6G and R123 on glass for several concentrations and rotation speeds in the ssp polarization combination. We see that the vibrational features remain essentially identical for both molecules and in all conditions, the rotation speed in particular does not deeply modify the spectra. One vibration mode 

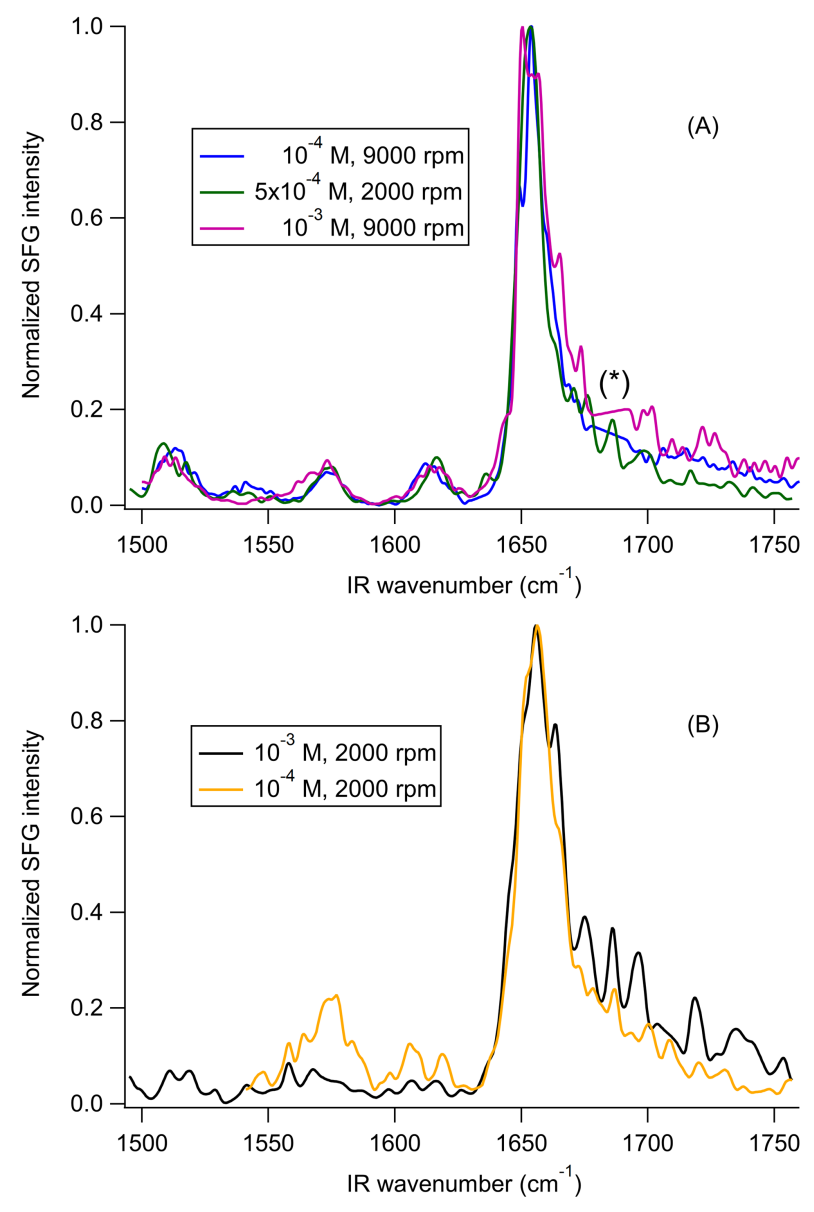

FIG. 3. Representative vibrational ssp SFG spectra of (A) R6G $\left(\lambda_{\text {vis }}=590 \mathrm{~nm}\right)$ and (B) R123 films $\left(\lambda_{\text {vis }}=560 \mathrm{~nm}\right)$. All intensities are normalized to 1 to ease comparison of the vibrational features. Remnants of water absorption still appear as dips. On some spectra, linear interpolation due to laser malfunction is indicated by $(*)$.

around $1655 \mathrm{~cm}^{-1}$ dominates all others $\left(1615 \mathrm{~cm}^{-1}, 1573 \mathrm{~cm}^{-1}\right.$ and $\left.1512 \mathrm{~cm}^{-1}\right)$ with a long tail extending on the high energy side. These modes are documented in the literature but we note that their intensity ratios differ from previously published data. ${ }^{30,31}$ This could be due to a different adsorption geometry, modifying the relative excitations of vibrational transition moments by IR light. The high energy tail may be attributed to interference with low intensity vibration modes above $1700 \mathrm{~cm}^{-1} \cdot 31,74,93$ Nonetheless, the main peak appears almost free from interference on the low energy side. It has been shown to be almost exclusively Franck-Condon active, contrary to the Herzberg-Teller active $1615 \mathrm{~cm}^{-1}$ mode. ${ }^{31}$ 

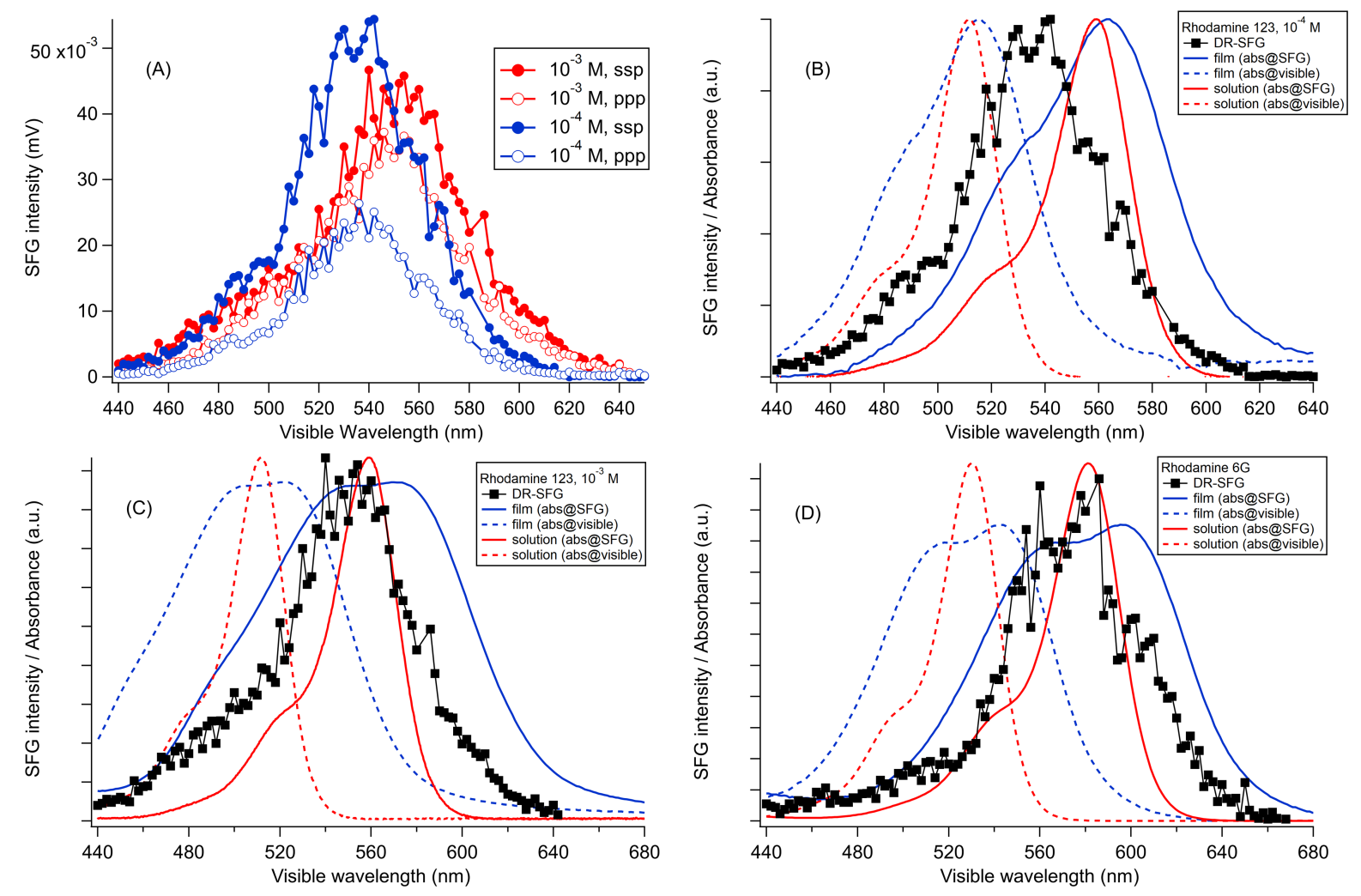

FIG. 4. (A) DR-SFG spectra recorded on the R123 films $\left(10^{-4} \mathrm{M}\right.$ in blue and $10^{-3} \mathrm{M}$ in red) for an IR wavenumber fixed at the $1655 \mathrm{~cm}^{-1}$ peak, as a function of the visible wavelength. Plain dots: ssp polarizations, open dots: ppp polarizations. (B)-(D) Comparison of the ssp DR-SFG response (black dots), absorbance in solution (red) and in the films (blue) at the visible (dotted lines) and

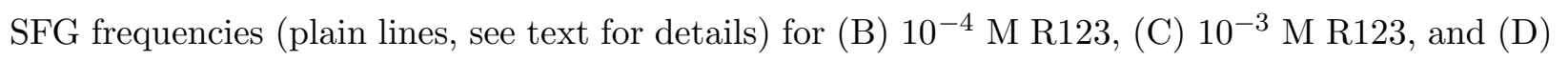
R6G.

\section{DR-SFG spectra of the rhodamine films}

In order to analyze the DR-SFG response in the visible range, we concentrate on the main vibration mode around $1655 \mathrm{~cm}^{-1}$. Its Franck-Condon nature and the low influence of interferences with the neighbouring peaks make it the ideal candidate for a comparison with the models described above. In the following, we fix the IR wavenumber to the value at peak maximum and record the SFG intensity as a function of the incoming visible wavelength tuned between $440 \mathrm{~nm}$ and $660 \mathrm{~nm}$ with a $2 \mathrm{~nm}$ step (Fig. 4). Several spectra were recorded sequentially, changing the probed point on the surface to avoid damaging the films. Points recorded for a visible wavelength around $582 \mathrm{~nm}$ were discarded as they produce SFG 
around $532 \mathrm{~nm}$, leading to detection saturated with stray light scattered from the doubled $\mathrm{YVO}_{4}$ source. We observed that the absolute intensities and ssp/ppp intensity ratios may change from point to point, the latter remaining between 1 and 2. An example of absolute comparison between ssp and ppp intensities for the R123 films is displayed on Fig. 4A, but the analysis below will focus on normalized spectral lineshapes rather than absolute intensities. We see that the DR-SFG intensities for R123 and R6G vary with the visible color, with a maximum in the green region, confirming previous observations for R6G and proving the double resonance. ${ }^{30,32}$ Fig. $4 \mathrm{~A}$ confirms an essential prediction of the theory, as the energy lineshape for a given film is found identical whatever the polarization combination. As shown in Ref. 74, the $D_{l}$ function is indeed common to all hyperpolarizability components and transfers to the macroscopic susceptibility components, leading to identical spectral lineshapes in the visible range for ssp and ppp polarization combinations as long as interference with a nonresonant and neighbouring peaks is minimal. In the following, we may thus use either ssp or ppp polarization combinations to model the energy dispersion of the DR-SFG excitation profiles. These profiles may be directly compared to the absorption properties of the solutions and the films, with resonance at the visible or SFG energies. For this purpose, we first draw in Fig. 4B-D the absorption spectra as a function of the visible wavelength. In order to check resonance between absorption processes and SFG wavelengths, we should draw on a separate graph absorbance and DR-SFG spectra as a function of the SFG wavelengths. For simplicity, we have chosen instead to add to Fig. 4B-D the absorbance spectra as a function of the visible wavelength, but redshifted by $1655 \mathrm{~cm}^{-1}$ to generate resonance with the SFG beam. For rhodamine 123 at $10^{-4} \mathrm{M}$, the DR-SFG resonance lies in the middle of the visible and SFG absorption resonances. As expected from the absorption spectra, the DR-SFG response varies with the concentration of the solutions used to prepare the films. For the $10^{-3} \mathrm{M}$ film, and for R6G, the DR-SFG profile appears to coincide with the high energy peak, attributed to $\mathrm{H}$-aggregates, in the film absorbance curve evaluated at the SFG frequency. From these curves, the DR-SFG profile overlaps more with the absorption features at the SFG energy than the visible one. However, it clearly appears that a simple interpretation straightforwardly deduced from the absorption curves is not possible here. 


\section{Modeling of the excitation spectra}

\section{J-aggregated film: $10^{-4}$ M Rhodamine 123}

In order to account for the experimental behaviours of the DR-SFG lineshapes, we apply the $\Phi$ function formalism outlined in Part II. We first focus on the $10^{-4} \mathrm{M}$ film because it appears that the absorption spectrum may be modeled by a single electronic excited state, corresponding to J-aggregated molecules. In a first step, the absorption spectrum of the film is divided by $\omega$ and the background subtracted to create the imaginary part of the $\Phi$ function (Eq. 4). The background has been shown to arise from the excitation of electronic transition below 400nm. ${ }^{85}$ A KK transformation (Eq. 5) is then performed to calculate the real part of $\Phi(\omega)$.

In a second step, we fit the imaginary part of the $\Phi(\omega)$ function of the $10^{-4} \mathrm{M}$ R123 film according to Eq. 6. Considering the high number of free parameters in the fit $\left(A_{0-0}, A_{\mathrm{vib}}\right.$, $\left.\omega_{0}, \omega_{c}, \Gamma_{0}, \Gamma_{\mathrm{vib}}, \sigma_{0}, \sigma_{\mathrm{vib}}\right)$, we have first started by fitting the R123 solution spectrum, then the film spectrum using the solution parameters as starting points. Curve fitting provided a very good agreement between experimental and fit points, thus validating the choice for the definition of the fit function (see Supplementary Material for details). To a given accuracy, the fits are not unique. This is not a problem as long as their imaginary parts accurately reproduce the experimental absorption curves, but it raises questions about the uniqueness of the real part deduced from the fits. We have checked that the latter is, whatever the fit parameters, identical to the real part deduced from KK transformation. As it is only a mathematical intermediate to evaluate the full $\Phi(\omega)$, this fitting procedure does not necessarily provide amplitude and width parameters having a direct physical interpretation. ${ }^{85}$

Through this procedure, we now have access to the real and imaginary part of the $\Phi$ function together (Fig. 5). As expected, the real part shows a derivative shape, and the imaginary part involves peaks, typical of a Lorentzian resonance. More surprisingly, when evaluating the features giving rise to the excitation profile $D_{l}$ (i.e. $\left.\Phi\left(\omega_{\mathrm{SFG}}\right)-\Phi\left(\omega_{\mathrm{vis}}\right)\right)$, the roles are reversed: the real part appears as a (negative) peak whereas the imaginary part shows a derivative shape. The excitation function may therefore be roughly seen as a quasi-Lorentzian resonance, inhomogeneously broadenend, centered close to the middle between visible and SFG wavelengths, with a $90^{\circ}$ phase delay as compared to the fundamental 


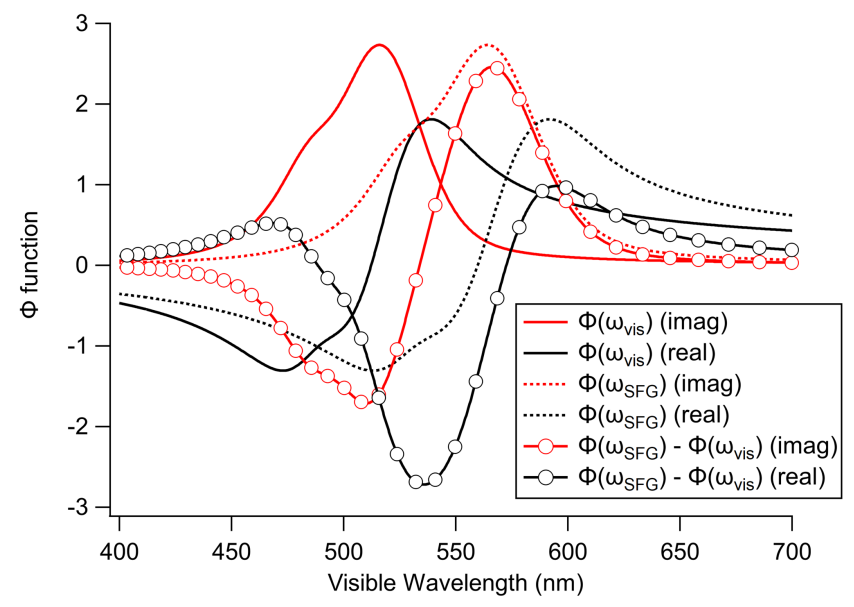

FIG. 5. Experimental $\Phi$ function of the R123 film $\left(10^{-4} \mathrm{M}\right)$ as a function of the visible wavelength (real part in black, imaginary part in red). For the evaluation of the DR-SFG excitation profile, the function is evaluated at the visible (plain lines) and SFG (dotted lines) wavelengths corresponding to the $1655 \mathrm{~cm}^{-1}$ peak. The differential function $\Phi\left(\omega_{\mathrm{SFG}}\right)-\Phi\left(\omega_{\mathrm{vis}}\right)$ appears as open circles.

processes. It may seem intriguing, but it results from the interference between the two $\Phi$ functions, with equal amplitudes and opposite signs, describing respectively the upward and downward contributions to the resonant Raman part of the DR-SFG process. We may establish a parallel with the Fano interferences between a continuum and discrete states, ${ }^{95}$ with the difference that all states here are discrete. They give rise to Fano profiles, which often have a derivative shape.

The dispersion of the excitation profile in the visible range only depends on the $\Phi$ terms in Eq. 3. From the experimentally determined $\Phi$ function, noting that Fresnel reflectivity on glass is essentially constant over the visible range, we may now easily calculate in Fig. 6 the normalized excitation profile (i.e. the energy lineshape) of the $1655 \mathrm{~cm}^{-1}$ mode, then simulate experimental intensity dispersion through $\left(\omega_{\mathrm{SFG}}\right)^{2}\left|\Phi\left(\omega_{\mathrm{SFG}}\right)-\Phi\left(\omega_{\mathrm{vis}}\right)\right|^{2}$, and compare it to the experimental points. We see that the simulations exactly match the original data (red points) on the high energy side, even accounting for the hump at the high energy peak foot, but extend too far on the low energy side. However, we observe that this low energy side is correctly accounted for when comparing the simulated curves to experimental points recorded on a different location on the film (orange squares). As a whole, the simulation agrees with the experimental points when the DR-SFG dispersions of the two locations on the film are taken into account together. In other words, the simulation represents an en- 


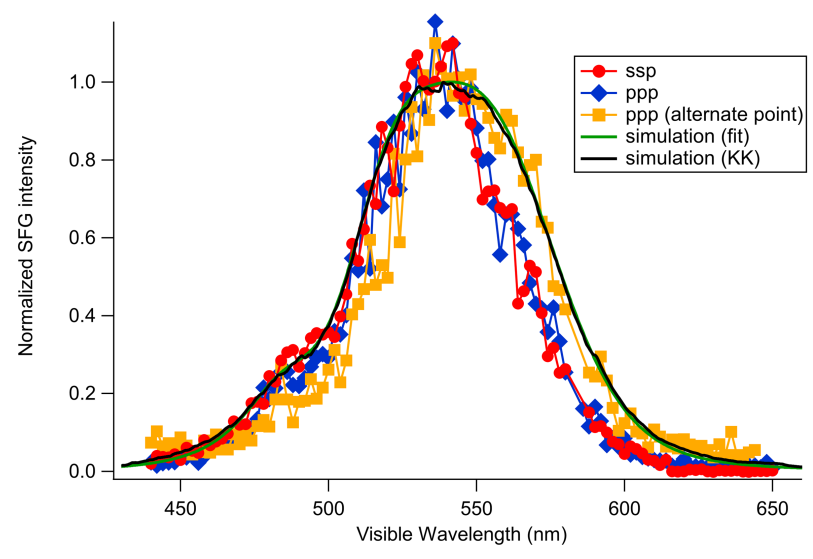

FIG. 6. Comparison of the simulated DR-SFG excitation profiles (plain lines) for the R123 film $\left(10^{-4} \mathrm{M}\right)$ at $1655 \mathrm{~cm}^{-1} \mathrm{IR}$ wavenumber using two methods for determining the $\Phi$ function (black: KK transformation, green: fit) with experimental ssp and ppp data recorded on two different locations on the film. All curves are normalized to unity in order to focus only on the lineshapes.

velope for the local experimental contributions. This is easily understood when comparing the experimental procedures in absorption and DR-SFG spectroscopies. Absorption spectra are recorded using a wide light beam which covers a large surface on the film. As the film is not homogeneous, the absorption spectrum integrates all the various local contributions into a broad spectrum, which appears in the end broader than the local resonant features. As the $\Phi$ function is deduced from this absorption spectrum, it also encompasses these heterogeneities and so does the simulated DR-SFG excitation profile. On the contrary, on the SFG set-up, we use small and focussed beams, generating SFG photons in the small area where the IR and visible beams overlap. The DR-SFG data are by essence local data, still integrating heterogeneities at the molecular scale, but not at the film scale. This explains why simulations appear broader than experimental point distributions, the full simulated width being recovered when integrating the SFG response over a large macroscopic surface of the film. This also explains why absorption spectrum in the film appears much broader than in solution.

The accurate simulation of the experimental data on Fig. 6 addresses several interesting conclusions. It shows that the transform method and direct use of the $\Phi$ function, deduced from in situ experimental absorption spectra, for the analysis of DR-SFG excitation profiles works perfectly well. This validates this methodology for the first time on DR-SFG. This 
also shows that the assumptions made in this study are indeed fulfilled: separability in DRSFG between molecular amplitudes and energy lineshapes; Franck-Condon nature of the

$1655 \mathrm{~cm}^{-1}$ mode; neglect of the higher order contributions to the excitation profile ${ }^{47}$ and of the interference effects with neighbouring vibration modes. The experimental shape in Fig. 4B is also now easily accounted for: the energy of the vibration mode is smaller than the width of the electronic resonance (in our case the full width, including inhomogeneous broadening), a situation which is known to produce a single peak in the excitation profile, ${ }^{74}$ located roughly halfway between the absorption maxima at visible and SFG frequencies. In addition, this proves that a single, inhomogeneously broadened, electronic resonance is enough to reproduce both the absorption and DR-SFG spectra. The main process at stake during film formation at this concentration is therefore J-aggregation, which appears before $\mathrm{H}$-aggregation starts. Finally, this study also proves that the films are indeed very heterogeneous, not only at the molecular scale but also at the macroscopic one. The film shows co-existence of various zones which differ much as for their aggregation properties, as evidenced by the experimental variabilities in their redshift values. Their optical responses sum up and eventually account for the different linewidths in the film and in solution. The competition between molecular adsorption and aggregation is a random process depending on several kinetic parameters, and it is not surprising that heterogeneity appears for low molecular concentration in solution.

\section{H- and J-aggregated films}

For the $10^{-3}$ M R123 and R6G films, the situation is complicated by the presence of both $\mathrm{H}$ - and J-aggregates together on the films, as evidenced by the splitting of the central peak on Fig. 2. We therefore apply the separation method proposed above. We start with the R6G film as the separation between $\mathrm{H}$ and $\mathrm{J}$ maxima is clearer on the absorption spectrum (Fig. 4D). This time, curve fitting is not just a trick for evaluating the real part from the knowledge of the imaginary part, it has to efficiently separate the contributions of the two electronic states. As an additional issue, the vibronic tail of the low energy resonance overlaps with the central peak of the high energy one, making it difficult to unambiguously separate them. As the superimposition of two $\operatorname{Im}[\Phi]$ functions in Eq. 10 results in a sum of four Faddeeva functions, and considering the weak amount of information conveyed by the 
structures of the absorption spectra, we limit the number of parameters by fixing identical structures (i.e. Lorentzian widths, gaussian widths, central peak over vibronic tail amplitude ratios) to the two $\Phi$ functions. This approximation is strengthened by the fact that both aggregation states rely on molecular interactions along two distinct geometries, but involving the same molecules in the same kind of environments. For these reasons, lifetimes and inhomogeneous broadenings of the two electronic states are essentially identical, as well as their average vibronic structures when considering that the aggregated molecules all share the same vibration modes as long as their geometries compare. The total fitting function is thus:

$$
\operatorname{Im}[\Phi(\omega)]=B_{H} \operatorname{Im}\left[\Phi_{J}\left(\omega-\omega_{H}\right)\right]+B_{J} \operatorname{Im}\left[\Phi_{J}(\omega)\right]
$$

where $\operatorname{Im}\left[\Phi_{J}(\omega)\right]$ has the form of Eq. 6. Even so, fitting is possible using very different sets of parameters, as several local minima may be found by the fitting algorithm. We present in Fig. 7A the decomposition of the total $\operatorname{Im}[\Phi]$ into $\mathrm{H}$ and $\mathrm{J}$ contributions with four different sets of parameters (details may be found in the Supplementary Material). A direct fitting gives rise to a marked vibronic tail, whereas subsequent fitting adjustments lead to configurations with a reduced or even absent tail. Comparison with the $10^{-4} \mathrm{M}$ R123 case tends to favour the intermediate situation, but we use all of them for the DR-SFG simulations.

We simulate the normalized SFG intensities as a function of the visible wavelength by evaluation of $\left(\omega_{\mathrm{SFG}}\right)^{2}\left|\Phi\left(\omega_{\mathrm{SFG}}\right)-\Phi\left(\omega_{\mathrm{vis}}\right)\right|^{2}$ and compare it to experimental ssp data. In order to understand the origin of the double resonance, we separately plot on Fig. 7B, the energy lineshapes of peaks $\mathrm{H}$ and J. As can be seen, the DR induced by the J peak alone is almost sufficient to recover the experimental energy profile. Although it is not possible to exactly reconstruct the complete profile with one of the four sets of parameters, we see that, for the three of them for which the vibronic tail is not overestimated, the essential features may be accounted for by the $\mathrm{J}$ resonance only, one of the fits even reconstructing the high energy tail below $520 \mathrm{~nm}$. We may conclude that DR-SFG is much more efficient here with the J-aggregates than the $\mathrm{H}$-aggregates. A minority contribution from $\Phi_{H}$ is also possible, with an impact mostly on the high energy tail, which could explain the discrepancies between experimental and simulated profiles in this region. However, it would also lead to a change in the high energy slope of the resonance peak (around $540 \mathrm{~nm}$ ). Given the accuracy of the $\mathrm{J}$ contribution in this region, the influence of the $\mathrm{H}$ contribution has to be small. We could 

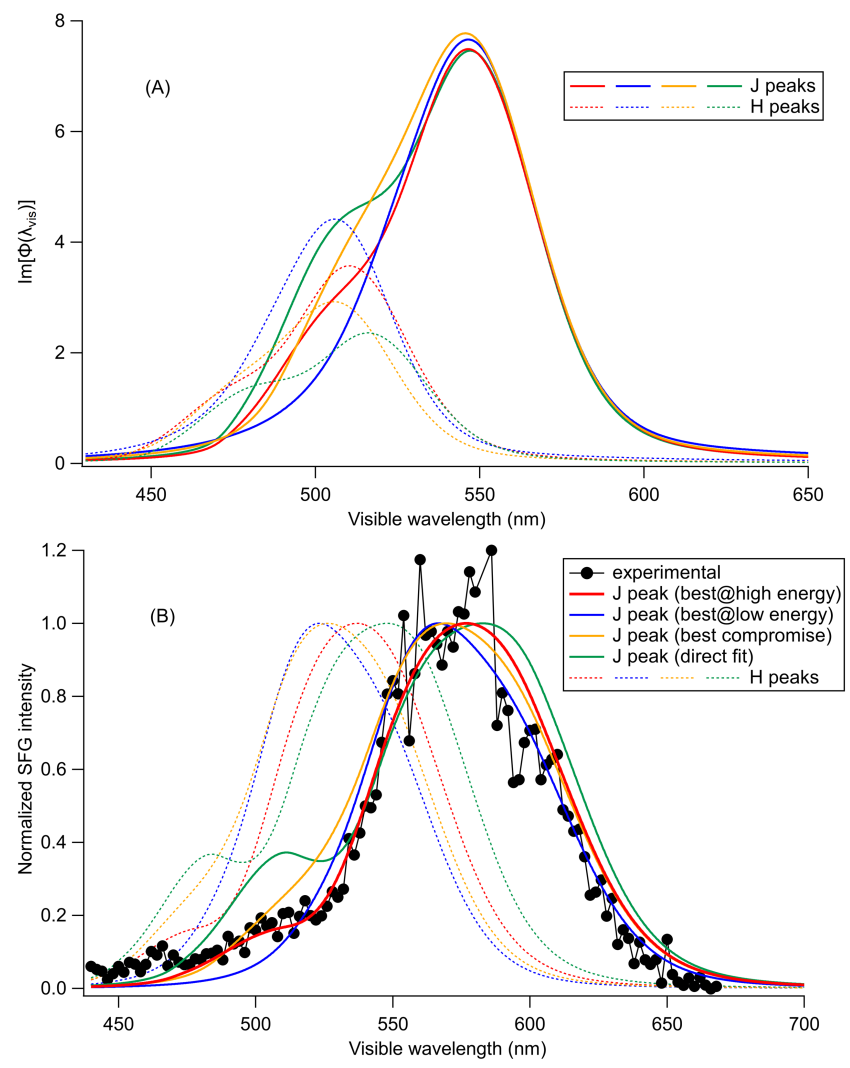

FIG. 7. (A) Imaginary parts of $\Phi\left(\omega_{\text {vis }}\right)$ (J peak: plain lines, H peak: dotted lines) for the R6G film at $1655 \mathrm{~cm}^{-1}$ IR wavenumber using four parameter sets for fitting the $\Phi$ function. (B) Comparison of the simulated DR-SFG excitation profiles with experimental ssp data for the same fitting parameters and peaks. All curves are normalized to unity in order to focus only on the lineshapes.

not find alternate experimental energy profiles by changing the probed location on the film (as was done with $10^{-4}$ M R123 in Fig. 6), which does not mean that such shifted profiles do not exist. They could also account for the discrepancies remaining on the low energy side of the resonance, giving even more credence to the interpretation in terms of $\mathrm{J}$ resonance only.

The same analysis was performed on the $10^{-3}$ M R123 film for the separation of $\mathrm{H}$ and J contributions to the total $\operatorname{Im}[\Phi]$ lineshape, and simulation of their normalized SFG profiles (Fig. 8). From the shape of the absorption spectrum (Fig. 4C), we see that the energy separation between the $\mathrm{H}$ and $\mathrm{J}$ absorption peaks is smaller than for R6G, indicating a lower aggregation state. However, the total width appears at least identical, which means that heterogeneity is greater in the R123 film, either locally (inhomogeneous broadening) or 

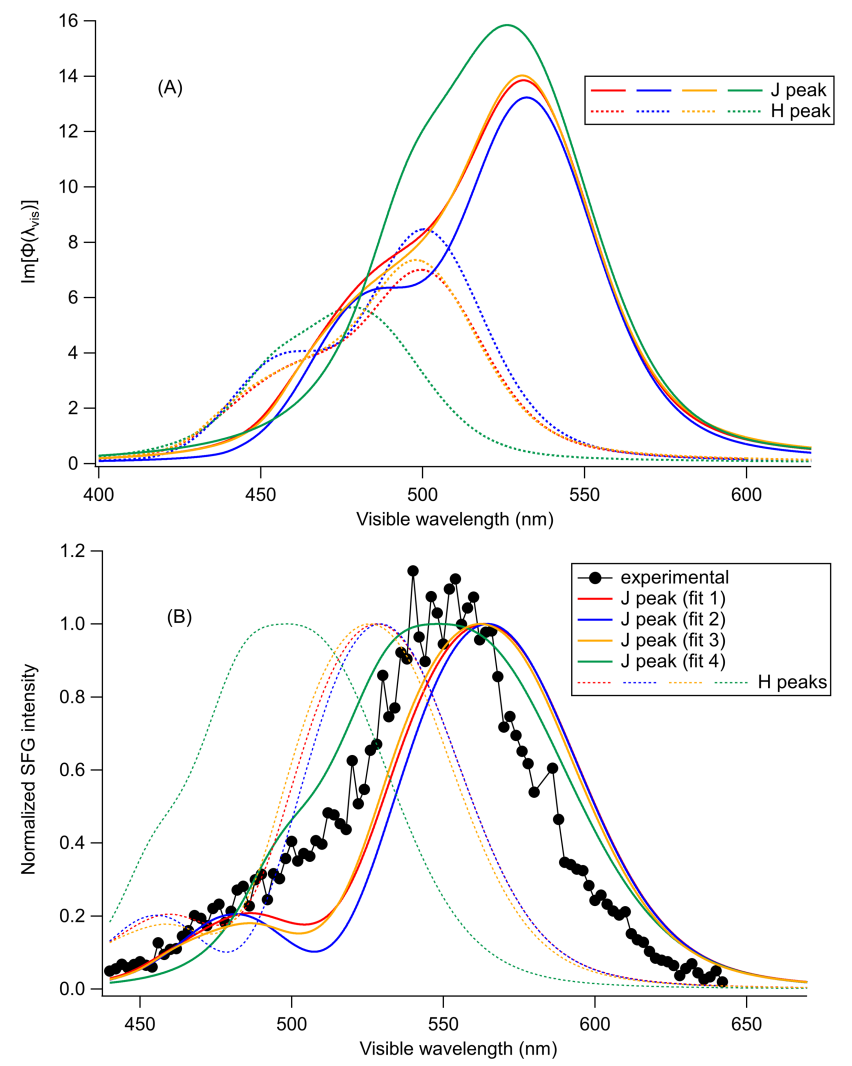

FIG. 8. (A) Imaginary parts of $\Phi\left(\omega_{\text {vis }}\right)$ (J peak: plain lines, H peak: dotted lines) for the $10^{-3} \mathrm{M}$ R123 film at $1655 \mathrm{~cm}^{-1}$ IR wavenumber using four parameter sets for fitting the $\Phi$ function. (B) Comparison of the simulated DR-SFG excitation profiles with experimental ssp data for the same fitting parameters and peaks. All curves are normalized to unity in order to focus only on the lineshapes.

macroscopically (different organizations throughout the film). In addition, the two maxima strongly overlap, making it difficult for the fitting algorithm to locate them accurately. As a consequence, the fitting step proved more difficult here, probably reaching the limits of the method. Still three sets of fitting parameters could be obtained in the same way as for R6G, with a rather marked vibronic tail. Looking for an alternate fit with less influence of the vibronic modes, we started with parameters inspired from the $10^{-4} \mathrm{M}$ film to get a fourth set of fitting parameters with a vibronic tail analogous to Fig. 5. We see in Fig. 8A that the fits strongly differ also by the positions of the $\mathrm{H}$ and $\mathrm{J}$ peaks, and the natures of their overlaps. The simulated SFG spectra (Fig. 8B) also show a dominant contribution from the J-aggregates. For the three first fits, there is an obvious shift in experimental 
peak position towards higher energies, which may hint at an important contribution from the $\mathrm{H}$-aggregates. For these fits, heterogeneity of the films alone cannot compensate for the incorrect position of the high energy slope of the central peak. For the fourth fit, however, the simulation results in an analogous situation as for $10^{-4} \mathrm{M}$ R123, with a very broad J peak encompassing all the experimental points on either side of the resonance, and a more blueshifted $\mathrm{H}$ peak. With this fit, all the double resonance, and the correct position of the maximum, may be accounted for by the J-aggregates alone. This hints at the pertinence of this analysis based on this fourth fit, even if no definite conclusion may be drawn without additional experimental evidence. Another point on the sample surface was probed, with no noticeable differences, as for the R6G film.

\section{DISCUSSION}

\section{A. Advantages and limitations of the method}

Analyzing DR-SFG data is an ongoing challenge. As discussed in Ref. 47, expressing the doubly resonant hyperpolarizability into Lorentzian sum-over-states or in integral form requires an explicit decomposition over the vibronic structure. The required parameters (vibronic energies, Huang-Rhys factors, coupling constants) start becoming accessible through quantum chemistry calculations. Practical implementation still leads, at some point, to simplifying the vibronic structure by neglecting some states or terms in the sums. In addition, these representations only provide theoretical and ideal simulations of the DR-SFG spectra, which do no take full account of the real vibronic structure (peak widths, sample heterogeneities, coexistence of various molecular states). The spirit of the transform method, on the other hand, is to directly insert the experimental ingredients (in our case the absorption spectrum) into the DR-SFG formalism by integrating all the vibronic parameters into the experimentally determined spectral overlap function $\Phi(\omega)$. The procedure is in fact rather simple: i) deduce the imaginary part of $\Phi(\omega)$ from the absorption spectrum $\mathrm{A}(\omega) / \omega$; ii) calculate the real part by a Kramers-Kronig transformation; iii) insert $\Phi$ into Eq. 2 or 3 to calculate the lineshape of the excitation profile. The main conclusion of the present study is the proof that this method works perfectly well to predict the spectral evolution of the DRSFG intensity in the visible range for a spin coated molecular film of organic chromophores 
involving very common deviations to the perfect picture: molecular aggregation, a variety of local environments leading to inhomogeneous broadening, and heterogenity in the film. For more complicated situations where two molecular aggregation states coexist, a fitting strategy is proposed which provides the essential required spectral structure but is of course limited by the accuracy of the experimental data used to build the $\Phi$ functions.

Application of the $\Phi$ function formalism relies on several hypotheses. First, we have

neglected terms arising from quadratic electron-vibration coupling. ${ }^{47,74}$ This does not imply that the vibration under study here is free from mode distortion or mode mixing. This rather means that either these effects may actually be neglected, or the formalism already partly includes them in its present form, which deserves future attention. On the other hand, the remaining discrepancies between simulations and experiments, in particular for the $10^{-3} \mathrm{M}$ R123 film at high energies, may be due, in addition to film heterogeneities, to the influence of these quadratic coupling terms. Second, this formalism only applies up to now to FranckCondon active modes, as the equations differ for Herzberg-Teller active modes. As the necessity to include Herzberg-Teller activity has been shown in some situations, especially for Franck-Condon inactive modes, ${ }^{31}$ the next step should be to develop the transform technique formalism for Herzberg-Teller terms and compare the visible dispersions for Franck-Condon and Herzberg-Teller modes. Third, we have neglected interference effects between vibration modes and focused on a single isolated mode in order to prove the reliability of the transform technique. Still, for a full vibrational DR-SFG spectrum comprising interfering modes, Eq. 2 allows to predict the visible dispersion in amplitude and in phase of the complex amplitude of each vibration mode. Consequently, it makes it possible to determine the vibrational phases and assign them during the fitting process, thus avoiding well-known issues of SFG curve fitting with free phase parameters. ${ }^{96}$

\section{B. Structure and DR-SFG activity of molecular aggregates}

Absorption data show that film formation on glass for rhodamine dyes from ethanol solutions follows a cumulative process. At low concentrations in solution, J-aggregates alone form on the glass surface, with a small redshift in absorption as compared to monomers. At higher concentrations, $\mathrm{H}$-aggregates appear when molecular packing and density raise, whereas the redshift of the J-aggregates increases. Molecular packing and film structures 
seem heterogeneous at all scales, in particular for the low concentrations where zones with different aggregation states macroscopically coexist in the film. This is confirmed by the DRSFG analysis. For the low concentration R123 film, the excitation profile of the $1655 \mathrm{~cm}^{-1}$ vibration mode is completely accounted for by a single electronic transition corresponding to J-aggregates, but a high macroscopic heterogeneity has been shown to contribute to the lineshape of its excitation profile. For the $5 \times 10^{-4} \mathrm{M}$ R6G film, J-aggregates account for almost all of the excitation profile in spite of the moderate to high content of $\mathrm{H}$-aggregate. Finally, for the high concentration R123 film, molecules involved in J-aggregates are also found to be more DR-SFG active than in $\mathrm{H}$-aggregates. Depending on the scenario considered, one fit leads to a very dominant $\mathrm{J}$ response, whereas the three other fits seem to favour mixed contributions. However, as the full response is the complex sum of $\mathrm{H}$ and $\mathrm{J}$ contributions to the excitation profiles, ${ }^{32}$ it is difficult here to quantify their respective weights (as was done in the past on isomeric mixtures for example ${ }^{60}$ ) because the fitting procedure cannot unambiguously separate them, giving rise to several plausible combinations. In summary, for all rhodamine films on glass, we show that aggregation dominates the molecular structure, but mostly J-aggregated molecules are DR-SFG active. As for the origins of the differences in DR-SFG activities between $\mathrm{H}$ - and J-aggregates, as the $1655 \mathrm{~cm}^{-1}$ mode is Franck-Condon

active, they lie in the $\mu_{g \leftarrow e}^{\alpha} \mu_{e \leftarrow g}^{\beta}\left(\frac{\partial \mu^{\gamma}}{\partial Q_{l}}\right)_{0} \Delta_{l}$ amplitudes of their respective excitation profiles. Absorption spectroscopy shows that both aggregates exhibit an in-plane electronic transition with transition dipole moments of the same order of magnitude. The difference therefore does not relate to the electronic transition dipole moments, as confirmed by the analogy in the excitation spectra for both ssp and ppp polarization combinations. The difference must lie in the $\Delta_{l}\left(\frac{\partial \mu^{\gamma}}{\partial Q_{l}}\right)_{0}$ product. This could be a geometric effect, caused by a more favourable orientation of the molecules in the J-aggregates, enhancing their $\left(\frac{\partial \mu^{z}}{\partial Q_{l}}\right)_{0}$ projections in the IR p-polarized hyperpolarizability combinations. On the other hand, aggregation may also induce a change in the multimer electronic states ${ }^{32,39,40,97,98}$ leading to a reduction in the $\Delta_{l}$ in the $\mathrm{H}$-aggregates as compared to J-aggregates.

\section{Comparison with literature spectra and interpretations}

It has long been wondered why, in the original publication on DR-SFG of a rhodamine 6G film, ${ }^{30}$ the experimental excitation spectra recorded in the visible range did not show all the 
structures predicted by the theory. In brief, the sum-over-states formulation ${ }^{47,72}$ essentially predicts two peaks in the excitation spectrum, corresponding to the resonance of the SFG and visible beams, respectively, with the electronic transition energy (or more precisely with $\hbar \omega_{0}^{e g}$ corresponding to the vibrationless transition energy ${ }^{47,48}$ ). They correspond to the two excitations described by $\Phi\left(\omega_{\text {vis }}\right)$ and $\Phi\left(\omega_{\mathrm{SFG}}\right)$ in Eq. 3. Experimentally, only one peak was observed, apparently matching a molecular electronic transition resonant with the SFG beam. The authors explained this single peak by an underestimation of the Lorentzian widths $\Gamma_{e g, \text { vib }}$ of the vibronic states (i.e. overestimation of their lifetimes). This amounts to damping most of the vibronic resonances in the simulations and in particular all of the second peak. In the light of the present study, we may deduce that this is part of the explanation, but there are other sources of inaccuracy of the theoretical description they used. For the definition of the resonant denominators of the vibronic Lorentzians, theoretical parameters were imposed and fixed $\left(\omega_{0}^{e g} ; \Gamma_{e g 0} ; \Gamma_{e g, v i b}\right)$ and the sum-over-states was limited to the vibration mode excited by the IR photon. This illustrates the main shortcoming of such theoretical descriptions as they do not incorporate the actual values of the required parameters. The exact value of $\omega_{0}^{e g}$ is not known as it does not correspond to the experimental absorption maximum. Molecular aggregation in the film induces experimental energy shifts but was not taken into account, whereas heterogeneities increase the widths (including that of the $0-0$ transition) through a distribution of all parameters values. It has also been shown ${ }^{74}$ that the double peak structure in the theoretical excitation profile of a DR vibration mode only exists when the vibrational energy is bigger than the full width of the electronic resonance $\left(2 \Gamma_{e g 0}\right)$. Excitation profiles therefore appear as single broad peaks in the case of low energy vibration modes, but also for high energy modes when the experimental width of the electronic transition grows, because of inhomogeneous broadening for example. This is illustrated here for the $10^{-4} \mathrm{M}$ R123 film. It can be checked that the absorption spectrum recorded in Ref. 30 is indeed as broad as for our R6G film, and exhibits a very Gaussian profile, hinting at a probable high inhomogeneous broadening. Although the molecular and film structures differ in our study and theirs, as evidenced by the lineshapes of adsorption and vibrational SFG spectra, we may still consider that the generation of the DR excitation profiles follows the same trends. This is confirmed by the fact that, in both publications, the DR-SFG excitation profiles overlap with the absorption spectrum at the SFG frequency. In fact, even though the film structures may 
differ, the experimental DR-SFG excitation profiles look very similar. The direct sum-overstates procedure does not take all these effects into account, which are naturally embedded in the transform technique through the $\Phi$ function, in particular as far as $\omega_{0}^{e g}, \Gamma_{e g 0}$ and $\Gamma_{e g \text {,vib }}$ parameters are concerned. This explains why we succeed here in accounting for the DRSFG excitation profiles. As a summary, the expected second peak of the excitation spectra in Ref. 30 was apparently absent because inhomogeneous broadening, film heterogeneity, molecular aggregation, differences in $\mathrm{H}$ - and J-aggregate DR activities, electronic frequency shifts, and natural widths were inaccurately, or not all, taken into account, and the merging of the two peaks into one broad maximum not correctly simulated.

\section{CONCLUSION}

The transform technique (TT) formalism is proposed as an alternative to the standard sum-over-states to analyze the doubly-resonant SFG spectra. Based on experimental data only, this formalism gets rid of all the unknown parameters of the sum-over-states, and requires no additional hypothesis on the vibronic modes, energies or widths. Here we have proven its efficiency even in complex cases to determine the energy dispersion of the excitation spectrum of Franck-Condon modes. This allows direct fitting of the spectrum as a function of $\omega_{\mathrm{IR}}$ while including appropriate amplitude and phase correction factors, and thus extract the relevant molecular parameters (IR activities, electronic transition dipole moments, normal mode coordinate shifts) from the fits.

In general, molecular interactions between organic chromophores in thin films will most of the time lead to modifications of the molecular optical properties, even below the monolayer threshold. The adsorption process on the substrate may also modify the molecular

electronic states, through charge transfer for example, as is well known in SERS. ${ }^{99}$ Finally, the amount of heterogeneity leading to inhomogeneous broadening can hardly be predicted when preparing a film by chemical routes. For these reasons, evaluation of the $\Phi$ function must rely on absorption spectra acquired in the same conditions as the DR-SFG spectra. This suggest that care should be taken when recording absorption data in situ: the spot size should be reduced to match the overlap size of the SFG process, and the same points on the surface should be probed by both techniques. In addition, to avoid solving puzzles on the complex $\Phi$ structure as we have done here for high concentration films, experimental 
procedures (substrate preparation method, molecular concentration, spin coating parameters) should be optimized in order to avoid aggregation, or at least control it to make it reproducible and free from mixing of aggregated states. This will ensure the robustness of the Kramers-Kronig step of the procedure. Finally, film preparation should also include a control step of macroscopic heterogeneities to validate or discard the samples. Within these constraints, we have shown that our formalism leads to a straightforward interpretation of DR-SFG spectroscopic data.

\section{SUPPLEMENTARY MATERIAL}

See Supplementary Material for details of the fitting procedure, curves and parameters.

\section{ACKNOWLEDGMENTS}

The collaboration between the University of Victoria and Université Paris-Saclay was supported by the French Embassy in Canada through the France-Canada Research Fund.

\section{DATA AVAILABILITY}

The data that support the findings of this study are available from the corresponding author upon reasonable request.

\section{REFERENCES}

${ }^{1}$ A. Hagfeldt, G. Boschloo, L. Sun, L. Kloo, and H. Pettersson, Chem. Rev. 110, 6595 (2010).

${ }^{2}$ A. Mishra, M. K. R. Fischer, and P. Bäuerle, Angew. Chem. Int. Ed. 48, 2474 (2009).

${ }^{3}$ A. Yella, H.-W. Lee, H. N. Tsao, C. Yi, A. K. Chandiran, M. K. Nazeeruddin, E. W.-G.

Diau, C.-Y. Yeh, S. M. Zakeeruddin, and M. Grätzel, Science 334, 629 (2011).

${ }^{4}$ S. Ye, A. Kathiravan, H. Hayashi, Y. Tong, Y. Infahsaeng, P. Chabera, T. Pascher, A. P. Yartsev, S. Isoda, H. Imahori, and V. Sundström, J. Phys. Chem. C 117, 6066 (2013).

${ }^{5}$ M. Urbani, M. Grätzel, M. K. Nazeeruddin, and T. Torres, Chem. Rev. 114, 12330 (2014).

${ }^{6}$ T. Higashino and H. Imahori, Dalton Trans. 44, 448 (2015). 
${ }^{7}$ L. Martín-Gomis, F. Fernández-Lázaro, and A. Sastre-Santos, J. Mater. Chem. A 2, 15672 (2014).

${ }^{8}$ H. N. Tsao, C. Yi, T. Moehl, J.-H. Yum, S. M. Zakeeruddin, M. K. Nazeeruddin, and M. Grätzel, ChemSusChem 4, 591 (2011).

${ }^{9}$ L. Kloo, Chem. Comm. 49, 6580 (2013).

${ }^{10}$ M. Liang and J. Chen, Chem. Soc. Rev. 42, 3453 (2013).

${ }^{11}$ G. Chen, H. Sasabe, T. Igarashi, Z. Hong, and J. Kido, J. Mater. Chem. A 3, 14517 (2015).

${ }^{12}$ A. K. Singh, A. Maibam, B. H. Javaregowda, R. Bisht, A. Kudlu, S. Krishnamurty, K. Krishnamoorthy, and J. Nithyanandhan, J. Phys. Chem. C 124, 18436 (2020).

${ }^{13}$ M. K. Nazeeruddin, P. Péchy, and M. Grätzel, Chem. Comm. 1997, 1705 (1997).

${ }^{14}$ M. Sánchez Carballo, M. Urbani, A. K. Chandiran, D. González-Rodríguez, P. Vázquez, M. Grätzel, M. K. Nazeeruddin, and T. Torres, Dalton Trans. 43, 15085 (2014).

${ }^{15}$ K. R. Mulhern, M. R. Detty, and D. F. Watson, J. Phys. Chem. C 115, 6010 (2011).

${ }^{16}$ G. Pepe, J. M. Cole, P. G. Waddell, and J. I. Perry, Mol. Syst. Des. Eng. 1, 416 (2016).

${ }^{17}$ M. Beija, C. A. M. Afonso, and J. M. G. Martinho, Chem. Soc. Rev. 38, 2410 (2009).

${ }^{18}$ Y. Kalachyova, D. Mares, V. Jerabek, K. Zaruba, P. Ulbrich, L. Lapcak, V. Svorcik, and O. Lyutakov, J. Phys. Chem. C 120, 10569 (2016).

${ }^{19}$ H. Watanabe, N. Hayazawa, Y. Inouye, and S. Kawata, J. Phys. Chem. B 109, 5012 (2005).

${ }^{20}$ C. B. Milojevich, D. W. Silverstein, L. Jensen, and J. P. Camden, J. Am. Chem. Soc. 133, 14590 (2011).

${ }^{21}$ C. B. Milojevich, D. W. Silverstein, L. Jensen, and J. P. Camden, J. Phys. Chem. C 117, 3046 (2013).

${ }^{22}$ H. K. Turley, Z. Hu, L. Jensen, and J. P. Camden, J. Phys. Chem. Lett. 8, 1819 (2017).

${ }^{23}$ C. H. Wang, O. Y.-H. Tai, Y. Wang, T.-H. Tsai, and N.-C. Chang, J. Chem. Phys. 122, 084509 (2005).

${ }^{24}$ P. Kaatz and D. P. Shelton, J. Opt. Soc. Am. B 16, 998 (1999).

${ }^{25}$ X. Qiu, X. Li, K. Niu, and S.-Y. Lee, J. Raman Spec. 42, 563 (2011).

${ }^{26}$ E. C. Le Ru, M. Meyer, and P. G. Etchegoin, J. Phys. Chem. B 110, 1944 (2006).

${ }^{27}$ M. D. Sonntag, J. M. Klingsporn, L. K. Garibay, J. M. Roberts, J. A. Dieringer, T. Seideman, K. A. Scheidt, L. Jensen, G. C. Schatz, and R. P. Van Duyne, J. Phys. Chem. C 
116, 478 (2012).

${ }^{28}$ G. Zengin, T. Gschneidtner, R. Verre, L. Shao, T. J. Antosiewicz, K. Moth-Poulsen, M. Käll, and T. Shegai, J. Phys. Chem. C 120, 20588 (2016).

${ }^{29}$ T. Kikteva, D. Star, Z. Zhao, T. L. Baisley, and G. W. Leach, J. Phys. Chem. B 103, 1124 (1999).

${ }^{30}$ M. Raschke, M. Hayashi, S. Lin, and Y. Shen, Chem. Phys. Lett. 359, 367 (2002).

${ }^{31}$ P. A. Weiss, D. W. Silverstein, and L. Jensen, J. Phys. Chem. Lett. 5, 329 (2014).

${ }^{32}$ S. Sengupta, L. Bromley III, and L. Velarde, J. Phys. Chem. C 121, 3424 (2017).

${ }^{33}$ B. Dick, A. Gierulski, G. Marowsky, and G. A. Reider, Appl. Phys. B 38, 107 (1985).

${ }^{34}$ R. Pandey, A. P. Moon, J. A. Bender, and S. T. Roberts, J. Phys. Chem. Lett. 7, 1060 (2016).

${ }^{35}$ L. Si and H. He, J. Phys. Chem. A 118, 3410 (2014).

${ }^{36}$ H. Imahori, S. Kang, H. Hayashi, M. Haruta, H. Kurata, S. Isoda, S. E. Canton, Y. Infahsaeng, A. Kathiravan, T. Pascher, P. Chábera, A. P. Yartsev, and V. Sundström, J. Phys. Chem. A 115, 3679 (2011).

${ }^{37}$ M. Chapman, M. Mullen, E. Novoa-Ortega, M. Alhasani, J. F. Elman, and W. B. Euler, J. Phys. Chem. C 120, 8289 (2016).

${ }^{38}$ J. R. Mann, M. K. Gannon, T. C. Fitzgibbons, M. R. Detty, and D. F. Watson, J. Phys. Chem. C 112, 13057 (2008).

${ }^{39}$ G. Chen, H. Sasabe, W. Lu, X.-F. Wang, J. Kido, Z. Hong, and Y. Yang, J. Mater. Chem. C 1, 6547 (2013).

${ }^{40}$ F. Milota, V. I. Prokhorenko, T. Mancal, H. von Berlepsch, O. Bixner, H. F. Kauffmann, and J. Hauer, J. Phys. Chem. A 117, 6007 (2013).

${ }^{41}$ S. M. Falke, C. A. Rozzi, D. Brida, M. Maiuri, M. Amato, E. Sommer, A. De Sio, A. Rubio, G. Cerullo, E. Molinari, and C. Lienau, Science 344, 1001 (2014).

${ }^{42}$ S. Ramakrishna, F. Willig, and V. May, Phys. Rev. B 62, R16330 (2000).

${ }^{43}$ A. Monti, C. F. A. Negre, V. S. Batista, L. G. C. Rego, H. J. M. de Groot, and F. Buda, J. Phys. Chem. Lett. 6, 2393 (2015).

${ }^{44}$ C. M. Johnson and E. Tyrode, Phys. Chem. Chem. Phys. 7, 2635 (2005).

${ }^{45}$ X. Zhuang, P. B. Miranda, D. Kim, and Y. R. Shen, Phys. Rev. B 59, 12632 (1999).

${ }^{46}$ E. H. G. Backus, A. Eichler, A. W. Kleyn, and M. Bonn, Science 310, 1790 (2005).

${ }^{47}$ B. Busson, J. Chem. Phys. 153, 174701 (2020). 
${ }^{48}$ J. Y. Huang and Y. R. Shen, Phys. Rev. A 49, 3973 (1994).

${ }^{49}$ P. Fischer and F. Hache, Chirality 17, 421 (2005).

${ }^{50}$ P. Fischer, F. W. Wise, and A. C. Albrecht, J. Phys. Chem. A 107, 8232 (2003).

${ }^{51}$ M. A. Belkin and Y. R. Shen, Phys. Rev. Lett. 91, 213907 (2003).

${ }^{52}$ R.-H. Zheng, D.-M. Chen, W.-M. Wei, T.-J. He, and F.-C. Liu, J. Phys. Chem. B 110, 4480 (2006).

${ }^{53}$ S. H. Han, N. Ji, M. A. Belkin, and Y. R. Shen, Phys. Rev. B 66, 165415 (2002).

${ }^{54}$ R.-H. Zheng, W.-M. Wei, Y.-Y. Jing, H. Liu, and Q. Shi, J. Phys. Chem. C 117, 11117 (2013).

${ }^{55}$ M. Okuno, D. Ishikawa, W. Nakanishi, K. Ariga, and T.-A. Ishibashi, J. Phys. Chem. C 121, 11241 (2017).

${ }^{56}$ L. Dreesen, C. Humbert, Y. Sartenaer, Y. Caudano, C. Volcke, A. A. Mani, A. Peremans, P. A. Thiry, S. Hanique, and J.-M. Frère, Langmuir 20, 7201 (2004).

${ }^{57}$ T. Maeda, T. Nagahara, M. Aida, and T.-A. Ishibashi, J. Raman Spec. 39, 1694 (2008).

${ }^{58}$ T. Nagahara, K. Kisoda, H. Harima, M. Aida, and T.-a. Ishibashi, J. Phys. Chem. B 113, 5098 (2009).

${ }^{59}$ T. Miyamae, E. Ito, Y. Noguchi, and H. Ishii, J. Phys. Chem. C 115, 9551 (2011).

${ }^{60}$ M. Raab, J. C. Becca, J. Heo, C.-K. Lim, A. Baev, L. Jensen, P. N. Prasad, and L. Velarde, J. Chem. Phys. 150, 114704 (2019).

${ }^{61}$ Q. Li, R. Hua, and K. C. Chou, J. Phys. Chem. B 112, 2315 (2008).

${ }^{62}$ T. Miyamae, K. Tsukagoshi, and W. Mizutani, Phys. Chem. Chem. Phys. 12, 14666 (2010).

${ }^{63}$ Y. Caudano, C. Silien, C. Humbert, L. Dreesen, A. Mani, A. Peremans, and P. Thiry, J. Electron Spectros. Relat. Phenomena 129, 139 (2003).

${ }^{64}$ K. C. Chou, S. Westerberg, Y. R. Shen, P. N. Ross, and G. A. Somorjai, Phys. Rev. B 69, 153413 (2004).

${ }^{65}$ B. Bozzini, L. D’Urzo, C. Mele, B. Busson, C. Humbert, and A. Tadjeddine, J. Phys. Chem. C 112, 11791 (2008).

${ }^{66}$ T. Miyamae, Y. Miyata, and H. Kataura, J. Phys. Chem. C 113, 15314 (2009).

${ }^{67}$ E. Kakudji, C. Silien, D. Lis, F. Cecchet, A. Nouri, P. A. Thiry, A. Peremans, and Y. Caudano, Phys. Stat. Sol. B 247, 1992 (2010).

${ }^{68}$ S. Yang, H. Noguchi, and K. Uosaki, J. Phys. Chem. C 119, 26056 (2015). 
${ }^{69}$ D. Elsenbeck, S. K. Das, and L. Velarde, Phys. Chem. Chem. Phys. 19, 18519 (2017).

${ }^{70}$ D. W. Silverstein and L. Jensen, J. Chem. Phys. 136, 064111 (2012).

${ }^{71}$ D. W. Silverstein and L. Jensen, J. Chem. Phys. 136, 064110 (2012).

${ }^{72}$ M. Hayashi, S. H. Lin, M. B. Raschke, and Y. R. Shen, J. Phys. Chem. A 106, 2271 (2002).

${ }^{73}$ J. C. Vallet, A. J. Boeglin, J. P. Lavoine, and A. A. Villaeys, Phys. Rev. A 53, 4508 (1996).

${ }^{74}$ B. Busson, J. Chem. Phys. 153, 174702 (2020).

${ }^{75}$ V. Hizhyakov and I. Tehver, Phys. status solidi 21, 755 (1967).

${ }^{76}$ D. L. Tonks and J. B. Page, Chem. Phys. Lett. 66, 449 (1979).

${ }^{77}$ J. Guthmuller and B. Champagne, J. Chem. Phys. 127, 164507 (2007).

${ }^{78}$ J. B. Page and D. L. Tonks, J. Chem. Phys. 75, 5694 (1981).

${ }^{79}$ J. Neugebauer and B. A. Hess, J. Chem. Phys. 120, 11564 (2004).

${ }^{80}$ L. Jensen, L. L. Zhao, J. Autschbach, and G. C. Schatz, J. Chem. Phys. 123, 174110 (2005).

${ }^{81}$ W. L. Peticolas and T. Rush, J. Comput. Chem. 16, 1261 (1995).

${ }^{82}$ T. W. Patapoff, P. Y. Turpin, and W. L. Peticolas, J. Phys. Chem. 90, 2347 (1986).

${ }^{83}$ T. I. Rush and W. L. Peticolas, J. Phys. Chem. 99, 14647 (1995).

${ }^{84}$ R. Kumble, T. S. Rush, M. E. Blackwood Jr, P. M. Kozlowski, and T. G. Spiro, J. Phys. Chem. B 102, 7280 (1998).

${ }^{85}$ A. Djorović, M. Meyer, B. L. Darby, and E. C. Le Ru, ACS Omega 2, 1804 (2017).

${ }^{86}$ L. Velarde and H. F. Wang, J. Chem. Phys. 139, 084204 (2013).

${ }^{87}$ R. J. Wells, J. Quant. Spec. Rad. Transf. 62, 29 (1999).

${ }^{88}$ L. Dalstein, C. Humbert, M. Ben Haddada, S. Boujday, G. Barbillon, and B. Busson, J. Phys. Chem. Lett. 10, 7706 (2019).

${ }^{89}$ I. Gordon, L. Rothman, C. Hill, R. Kochanov, Y. Tan, P. Bernath, M. Birk, V. Boudon, A. Campargue, K. Chance, B. Drouin, J.-M. Flaud, R. Gamache, J. Hodges, D. Jacquemart, V. Perevalov, A. Perrin, K. Shine, M.-A. Smith, J. Tennyson, G. Toon, H. Tran, V. Tyuterev, A. Barbe, A. Császár, V. Devi, T. Furtenbacher, J. Harrison, J.-M. Hartmann, A. Jolly, T. Johnson, T. Karman, I. Kleiner, A. Kyuberis, J. Loos, O. Lyulin, S. Massie, S. Mikhailenko, N. Moazzen-Ahmadi, H. Müller, O. Naumenko, A. Nikitin, O. Polyansky, M. Rey, M. Rotger, S. Sharpe, K. Sung, E. Starikova, S. Tashkun, J. V. 
Auwera, G. Wagner, J. Wilzewski, P. Wcisło, S. Yu, and E. Zak, J. Quant. Spectrosc. Radiat. Transf. 203, 3 (2017).

${ }^{90}$ E. Novoa-Ortega, M. Dubnicka, and W. B. Euler, J. Phys. Chem. C 124, 16058 (2020).

${ }^{91}$ J. Bujdák and N. Iyi, J. Phys. Chem. B 109, 4608 (2005).

${ }^{92}$ P. R. Ojeda, I. A. K. Amashta, J. R. Ochoa, and I. L. Arbeloa, J. Chem. Soc., Faraday Trans. 2 84, 1 (1988).

${ }^{93}$ M. Majoube and M. Henry, Spectrochim. Acta Part A 47, 1459 (1991).

${ }^{94}$ L. Jensen and G. C. Schatz, J. Phys. Chem. A 110, 5973 (2006).

${ }^{95}$ U. Fano, Phys. Rev. 124, 1866 (1961).

${ }^{96}$ B. Busson and A. Tadjeddine, J. Phys. Chem. C 113, 21895 (2009).

${ }^{97}$ A. Mishra, R. K. Behera, P. K. Behera, B. K. Mishra, and G. B. Behera, Chem. Rev. 100, $1973(2000)$.

${ }^{98}$ G. Zajac, A. Kaczor, A. Pallares Zazo, J. Mlynarski, M. Dudek, and M. Baranska, J. Phys. Chem. B 120, 4028 (2016).

${ }^{99}$ K. Ikeda, S. Suzuki, and K. Uosaki, Nano Lett. 11, 1716 (2011). 